\title{
Is the concept of the non-Hermitian effective Hamiltonian relevant in the case of potential scattering?
}

\author{
Dmitry V. Savin ${ }^{1,2}$, Valentin V. Sokolov ${ }^{2}$ and Hans-Jürgen Sommers ${ }^{1}$ \\ ${ }^{1}$ Fachbereich Physik, Universität-GH Essen, 45117 Essen, Germany \\ ${ }^{2}$ Budker Institute of Nuclear Physics, 630090 Novosibirsk, Russia
}

(Received 11 June 2002; Published 26 February 2003 in: Phys. Rev. E 67, 026215 (2003))

\begin{abstract}
We examine the notion and properties of the non-Hermitian effective Hamiltonian of an unstable system using as an example potential resonance scattering with a fixed angular momentum. We present a consistent self-adjoint formulation of the problem of scattering on a finite-range potential, which is based on separation of the configuration space into two segments, internal and external. The scattering amplitude is expressed in terms of the resolvent of a non-Hermitian operator $\mathcal{H}$. The explicit form of this operator depends on both the radius of separation and the boundary conditions at this place, which can be chosen in many different ways. We discuss this freedom and show explicitly that the physical scattering amplitude is, nevertheless, unique although not all choices are equally adequate from the physical point of view.

The energy-dependent operator $\mathcal{H}$ should not be confused with the non-Hermitian effective Hamiltonian $\mathcal{H}_{\text {eff }}$ which is usually exploited to describe interference of overlapping resonances. We note that the simple Breit-Wigner approximation is as a rule valid for any individual resonance in the case of few-channel scattering on a flat billiard-like cavity, leaving no room for non-trivial $\mathcal{H}_{\text {eff }}$ to appear. The physics is appreciably richer in the case of an open chain of $L$ connected similar cavities whose spectrum has a band structure. For a fixed band of $L$ overlapping resonances, the smooth energy dependence of $\mathcal{H}$ can be ignored so that the constant $L \times L$ submatrix $\mathcal{H}_{\text {eff }}$ approximately describes the time evolution of the chain in the energy domain of the band and the complex eigenvalues of $\mathcal{H}_{\text {eff }}$ define the energies and widths of the resonances. We apply the developed formalism to the problem of a chain of $L \delta$ barriers whose solution is also found independently in a closed form. We construct $\mathcal{H}_{\text {eff }}$ for the two commonly considered types of the boundary conditions (Neumann and Dirichlet) for the internal motion. Although the final results are in perfect coincidence, somewhat different physical patterns arise of the trend of the system with growing openness. Formation in the outer well of a short-lived doorway state shifted in the energy is explicitly demonstrated together with the appearance of $L-1$ long-lived states trapped in the inner part of the chain.
\end{abstract}

PACS numbers: 05.60.Gg, 03.65.Nk, 24.30.-v, 73.23.-b

\section{INTRODUCTION}

To the best of our knowledge, the concept of the nonHermitian effective Hamiltonian appeared first in Feshbach's papers 1, 2] in connection with the general theory of resonance nuclear reactions $[3,4]$ and, independently, in Livšic's study of open systems [5, 6]. A typical atomic nucleus forms near an excitation energy $E$ above the threshold of nucleon emission a rich set of long-lived compound states with a very dense energy spectrum. For relatively small excitation energies, these states manifest themselves as narrow isolated resonances in collisions of nucleons and target nuclei. The sharp energy dependence of the corresponding cross sections near a given resonance is described by the universal Breit-Wigner formula. Any smoother variations can be ignored in the domain of an isolated resonance. At higher $E$, the resonance states begin to overlap and strongly interfere. Nevertheless, one can still neglect the smooth energy dependence within a group of close interfering resonances. In this approximation, the propagation of the unstable system created on the intermediate stage of the collision is characterized by the resolvent of an energy-independent non-Hermitian matrix [7]. The latter describes in the time picture the irreversible evolution of the excited intermediate state and can therefore be interpreted as the effective Hamiltonian $\mathcal{H}_{\text {eff }}$ whose anti-Hermitian part, originating from on-shell self-energy contributions, is responsible for decays into open channels. The complex eigenvalues of the effective Hamiltonian determine the energies and widths of the resonances. The method of the effective Hamiltonian proved to be a success for describing many important resonance phenomena, especially in the context of chaotic scattering 8 , 9, 10, 11, 12, 13, 14.

It must be stressed that, similar to the Breit-Wigner formula, the notion of the effective Hamiltonian is local in energy although the general approaches [3, 4] work in much wider energy intervals where smooth variations become important and should already be taken into account. For the very concept of the effective Hamiltonian to be consistent, the scales of resonant and smooth variations must be appreciably different. Otherwise the resonance behavior of scattering amplitudes will be distorted or even completely destroyed. The smooth dependence influences the background phases as well as the parameters of the resonances situated in different energy domains. Such effects cannot be described by simply enlarging the dimension of the matrix of the effective Hamiltonian. Instead, a large energy-dependent matrix $\mathcal{H}(E)$ emerges whose simple interpretation as a time-shift 
operator is no longer valid. There is, generally, no oneto-one correspondence between the complex energies of resonance states on the one hand and the $E$-dependent eigenvalues of this large matrix on the other.

The current intensive studies, theoretical as well as experimental, of chaotic scattering of a particle by an open two-dimensional cavity (see, e.g., 15] and references therein) renewed interest in the Hamiltonian approach to the resonance scattering theory and, in particular, to the concept and properties of the effective non-Hermitian Hamiltonian 16, 17, 18. Although the scattering processes considered are purely potential, the theory is formulated in close analogy with the formalism developed in 1, 2, 3, 4] for nuclear collisions. The configuration space is divided into two parts, internal and external. The Feshbach projection technique is employed to express the scattering matrix in the specific form that explicitly reveals the sharp resonant energy dependence while all smoother variations show up only indirectly via changes of the matrix elements. Related is the problem of electromagnetic field quantization in open optical cavities [19].

While the probability amplitudes of the physical processes are fixed unambiguously, the projection procedure exploited is not unique. There exists a rather wide freedom in choosing the surface of separation as well as the boundary conditions (BCs) on this surface. Therefore, the amplitudes of interest are expressed in terms of quantities that depend on the details of the formalism. The independence of the final results of the calculations is not, as a rule, directly seen. Therefore, a certain caution is necessary to avoid incorrect assertions. An unexpected dependence on the (auxiliary) boundary condition at the cavity-lead interface was found, for example, in [17]. Moreover, poor agreement with numerically calculated exact poles of the $S$ matrix was revealed for poles distant from the real axis. These points, partly attributed by the authors to numerical limitations, require further clarification. Additional physical arguments should sometimes be involved to reasonably restrict the freedom.

It is important to recognize that the density of levels in billiard-like cavities is much lower than that of the manybody nuclear systems. Actually, strong overlap and interference of different resonance states are not, as a rule, possible in the single two-dimensional cavities ordinarily considered [17]. The simple Breit-Wigner approximation is usually sufficient in this case for any individual resonance (see, e.g., 20, 21]). Noticeable interference can occur only in the rare event of accidental near degeneracy of resonances. Experimental observation of such an interference of few resonances in an open microwave cavity has recently been reported in 22. Stronger overlap and a non trivial effective Hamiltonian matrix can, however, appear when open chains of a number of similar potential wells connected to each other are considered. The energy spectrum has a band structure in this case with much denser spectrum within a given band. A schematic model of such a kind was considered in [23]. A similar example was also investigated in [13] in the framework of graph theory [24]. Some general aspects of scattering in periodic structures have also been considered in [25].

In this paper we examine the notion of the effective Hamiltonian in potential scattering. The questions we are concerned with do not depend on the regular or irregular character of the motion. Thus, we restrict ourselves to the simplest case of the single-channel $s$-wave scattering. The extension to higher partial waves is straightforward. A consistent self-adjoint formulation of the problem of scattering on a finite-size potential in terms of the internal and external subproblems is presented in Sec. II. The (one-dimensional) $S$ matrix is expressed in terms of a non-Hermitian energy-dependent operator $\mathcal{H}$ whose form, together with the form of the associated $R$ function (Sec. III), depends on the $\mathrm{BC}$ and the radius $a$ of separation in the configuration space. Different BCs define different representations of the internal and external parts of the (unique) scattering wave function. The role of the fictitious direct reflection at the separation point $a$ is also discussed.

In Sec. IV the exactly solvable open Kronig-Penney model is considered as an example of potential scattering with interfering resonances. Closed expressions are found for both scattering $S(k)$ and staying-wave $K(k)$ functions as well as Wigner's function $R(k)$. Their analytical properties in the complex $k$ and energy planes are analyzed in detail. Hereafter we compare these exact results with those obtained in the framework of the projection formalism of the previous sections. We analyze two typical choices of the BCs for the internal motion: the cases of Neumann and Dirichlet BCs. In the second case, the internal problem corresponds to a closed counterpart of the system under consideration that allows one to follow the changes of the motion due to the interference of resonances when the openness grows. The latter is in line with numerous applications considered in the literature. Non-Hermitian effective Hamiltonians for a fixed band of resonance states are built up in both cases. We summarize our main findings in the concluding Sec. V.

\section{SEPARATION OF THE HILBERT SPACE}

The radial motion in the $s$-wave scattering is described by the Schrödinger equation (we use the units $\hbar^{2} / 2 m=1$ throughout the paper)

$$
\left(-\frac{d^{2}}{d r^{2}}+U(r)\right) \chi(r)=k^{2} \chi(r)
$$

with the boundary condition $\chi(0)=0$ at the origin. We use below the specific method which, basically, goes back to Bloch's paper [26] (see also 27]). However, we present a derivation which leads to interrelated boundary problems for internal and external regions. Making use of Heaviside's step function one can decompose the wave 
function into internal and external parts as follows

$$
\chi(r)=u(r) \theta(a-r)+\phi(r) \theta(r-a),
$$

where the functions $u$ and $\phi$ are supposed to be continuous at the point $a$ together with their two first derivatives. The partition radius $a$ can be chosen arbitrarily; we suggest only that $U(r>a) \equiv 0$. The second derivative of the function (2) reads

$$
\begin{gathered}
\chi^{\prime \prime}(r)=u^{\prime \prime}(r) \theta(a-r)+\phi^{\prime \prime}(r) \theta(r-a)+ \\
{\left[\phi^{\prime}(a)-u^{\prime}(a)\right] \delta(r-a)-[u(a)-\phi(a)] \delta^{\prime}(r-a) .}
\end{gathered}
$$

When substituting this expression into Eq. (11), there exists a freedom in attributing the local terms in (3) to the internal or external regions. One possible choice is to define the singular functions as $\delta\left(r-a_{-}\right) \equiv\left\langle r \mid a_{-}\right\rangle=\left\langle a_{-} \mid r\right\rangle$ and $\delta^{\prime}\left(r-a_{+}\right)=-\frac{d}{d a_{+}} \delta\left(r-a_{+}\right) \equiv-\left\langle r \mid a_{+}\right\rangle^{\prime}=-\left\langle\left. a_{+}\right|^{\prime} r\right\rangle$, where $a_{ \pm}=a \pm 0$, thus considering the first as belonging to the internal subspace and the second to the external one, respectively.

With such definitions, we can represent (11) in the form

$$
\left(\begin{array}{cc}
H_{\mathrm{int}}^{(\mathrm{N})} & V \\
V^{\dagger} & H_{\mathrm{ext}}^{(\mathrm{D})}
\end{array}\right)\left(\begin{array}{c}
u \\
\phi
\end{array}\right)=k^{2}\left(\begin{array}{c}
u \\
\phi
\end{array}\right),
$$

with the following entries:

$$
\begin{gathered}
H_{\mathrm{int}}^{(\mathrm{N})}=\hat{K}+U+\left|a_{-}\right\rangle\left\langle\left. a_{-}\right|^{\prime},\right. \\
H_{\mathrm{ext}}^{(\mathrm{D})}=\hat{K}+\left|a_{+}\right\rangle^{\prime}\left\langle a_{+}\right|, \\
V=-\left|a_{-}\right\rangle\left\langle\left. a_{+}\right|^{\prime}, \quad V^{\dagger}=-\mid a_{+}\right\rangle^{\prime}\left\langle a_{-}\right|,
\end{gathered}
$$

where $\hat{K}$ stands for the kinetic energy operator. In particular, in the coordinate representation the matrix elements of the entries take the form

$$
\begin{aligned}
\left\langle r\left|H_{\mathrm{int}}^{(\mathrm{N})}\right| r^{\prime}\right\rangle= & {\left[-\frac{d^{2}}{d r^{2}}+U(r)\right] \delta\left(r-r^{\prime}\right) } \\
& -\delta\left(r-a_{-}\right) \delta^{\prime}\left(r^{\prime}-a_{-}\right), \\
\left\langle r\left|H_{\mathrm{ext}}^{(\mathrm{D})}\right| r^{\prime}\right\rangle= & -\frac{d^{2}}{d r^{2}} \delta\left(r-r^{\prime}\right) \\
& -\delta^{\prime}\left(r-a_{+}\right) \delta\left(r^{\prime}-a_{+}\right), \\
\left\langle r|V| r^{\prime}\right\rangle= & \left\langle r^{\prime}\left|V^{\dagger}\right| r\right\rangle=\delta\left(r-a_{-}\right) \delta^{\prime}\left(r^{\prime}-a_{+}\right) .
\end{aligned}
$$

The presence of the singular terms assures Hermiticity of the operators $H_{\text {int }}$ and $H_{\text {ext }}$ in contrast to $\hat{K}$ alone [26]. The relations $\left\langle u_{1} \mid H_{\mathrm{int}} u_{2}\right\rangle=\left\langle H_{\mathrm{int}} u_{1} \mid u_{2}\right\rangle$, etc., can be easily checked by means of partial integrations. The singular operators on the boundary provide the boundary conditions, the first one Neumann for the internal region, the second one Dirichlet for the external region. Equation (3) implies that both BCs are interrelated. This also leads to the adjointness of the coupling operators $V$ and $V^{\dagger}$. The Dirichlet boundary operator is essentially different from that of Neumann type and cannot be produced by the boundary operators as used in [26].

The range of operators in Eq. (6) within the Hilbert space is defined by the functions on which the singular terms vanish. This requirement fixes the $\mathrm{BCs}$ at the point of separation $a$. The full Hilbert space is a direct sum of the space spanned by the eigenvectors of the internal problem $0<r<a$ with Neumann BC,

$$
\left(-\frac{d^{2}}{d r^{2}}+U(r)\right) u_{n}^{0}(r)=\varepsilon_{n}^{(\mathrm{N})} u_{n}^{0}(r), \quad u_{n}^{0^{\prime}}(a)=0
$$

and that spanned by the eigenvectors of the external problem $r>a$ with Dirichlet BC,

$$
-\frac{d^{2}}{d r^{2}} \phi_{k}^{0}(r)=k^{2} \phi_{k}^{0}(r), \quad \phi_{k}^{0}(a)=0 ; k \geq 0 .
$$

The type of BC is explicitly indicated by the corresponding superscripts in Eqs. (44)-(6). As usual, we assume normalization conditions

$$
\begin{array}{r}
\int_{0}^{a} u_{n}^{0}(x) u_{m}^{0}(x) d x=\delta_{n m}, \\
\int_{a}^{\infty} \phi_{k}^{0}(x) \phi_{k^{\prime}}^{0}(x) d x=\delta\left(k-k^{\prime}\right) .
\end{array}
$$

in the discrete and continuous spectra, respectively. The external function

$$
\phi_{k}^{0}(r)=\sqrt{\frac{2}{\pi}} \sin [k(r-a)] \propto e^{-i k r}-e^{-2 i k a} e^{i k r}
$$

describes a wave fully reflected at the point $a$, the corresponding $S$ function being equal to $S_{0}^{(\mathrm{D})}(k)=e^{-2 i k a}$.

One can also proceed in the opposite way and ascribe the terms with $\delta^{\prime}$ and with $\delta$ to the internal and external regions, respectively. In this case, the entries in Eq. (4) read

$$
\begin{gathered}
H_{\mathrm{int}}^{(\mathrm{D})}=\hat{K}+U-\left|a_{-}\right\rangle^{\prime}\left\langle a_{-}\right|, \\
H_{\mathrm{ext}}^{(\mathrm{N})}=\hat{K}-\left|a_{+}\right\rangle\left\langle\left. a_{+}\right|^{\prime},\right. \\
\tilde{V}=\left|a_{-}\right\rangle^{\prime}\left\langle a_{+}\left|, \quad \tilde{V}^{\dagger}=\right| a_{+}\right\rangle\left\langle\left. a_{-}\right|^{\prime} .\right.
\end{gathered}
$$

That corresponds to the nonperturbed internal problem with Dirichlet BC $v_{n}^{0}(a)=0$, when the external one has Neumann $\mathrm{BC} \varphi_{k}^{0^{\prime}}(a)=0$. The latter results in an additional shift by $\pi / 2$ of the reflection phase at the separation point $a: S_{0}^{(\mathrm{N})}(k)=-S_{0}^{(\mathrm{D})}(k)=e^{-2 i(k a+\pi / 2)}$.

The interplay of the internal and external motions due to the off-diagonal elements $V$, (5]c), distorts the outer waves $\phi$. From the upper row in Eq. (4) we find for all $k^{2} \neq \varepsilon_{n}, n=1,2, \ldots$

$$
u=\frac{1}{k^{2}-H_{\mathrm{int}}} V \phi \equiv G_{\mathrm{int}} V \phi .
$$

Here $G_{\text {int }}$ is the resolvent operator for the internal problem. Then, the lower row transforms into

$$
\left(k^{2}-H_{\mathrm{ext}}-V^{\dagger} G_{\mathrm{int}} V\right) \phi=0,
$$

or, in the position representation,

$$
\begin{gathered}
\left(k^{2}+\frac{d^{2}}{d r^{2}}\right) \phi(r)+\delta^{\prime}\left(r-a_{+}\right)\left[\phi(a)+G_{\mathrm{int}}^{(\mathrm{N})}(a, a) \phi^{\prime}(a)\right]=0, \\
\left(k^{2}+\frac{d^{2}}{d r^{2}}\right) \phi(r)-\delta\left(r-a_{+}\right)\left[\phi^{\prime}(a)-G_{\mathrm{int}}^{(\mathrm{D})}(a, a) \phi(a)\right]=0
\end{gathered}
$$


for the two cases considered. This yields change of the $\mathrm{BCs}$ of the external part of the exact wave function to

$$
\begin{gathered}
\phi(a)+G_{\mathrm{int}}^{(\mathrm{N})}(a, a) \phi^{\prime}(a)=0, \\
\phi^{\prime}(a)-G_{\mathrm{int}}^{(\mathrm{D})^{\prime \prime}}(a, a) \phi(a)=0,
\end{gathered}
$$

where the shorthand

$$
\left.G_{\text {int }}^{(\mathrm{D})^{\prime \prime}}(a, a) \equiv \frac{\partial^{2}}{\partial r \partial r^{\prime}} G_{\text {int }}^{(\mathrm{D})}\left(r, r^{\prime}\right)\right|_{r, r^{\prime} \rightarrow a}
$$

has been used. Let us note that Eq. 14a is the conventional boundary condition in the Wigner-Eisenbud $R$ matrix theory of resonance nuclear reactions [4, 28].

\section{III. $S-$ AND $R$-FUNCTIONS}

In the external region $r>a$, where the potential vanishes identically, the wave function has the form $\phi(r)=$ const $\left[e^{-i k r}-S(k) e^{i k r}\right]$. Therefore, the conditions (14) obtained above allow us to express the function

$$
S(k) \equiv e^{2 i \delta(k)}=\frac{1+i k \phi(a) / \phi^{\prime}(a)}{1-i k \phi(a) / \phi^{\prime}(a)} e^{-2 i k a}
$$

in terms of the internal Green's functions,

$$
\begin{aligned}
S(k) & =\frac{1-i k G_{\mathrm{int}}^{(\mathrm{N})}(a, a)}{1+i k G_{\mathrm{int}}^{(\mathrm{N})}(a, a)} S_{0}^{(\mathrm{D})}(k) \\
& =\frac{1-(i / k) G_{\mathrm{int}}^{(\mathrm{D})^{\prime \prime}}(a, a)}{1+(i / k) G_{\mathrm{int}}^{(\mathrm{D})^{\prime \prime}}(a, a)} S_{0}^{(\mathrm{N})}(k) .
\end{aligned}
$$

Both expressions are exact and equivalent to each other. The only difference is that they are written in different complete bases in the full Hilbert space. The merits as well as limitations of each of these representations will be discussed below.

We define further the phase shifts $\delta^{(\mathrm{N}, \mathrm{D})}(k)$ due to the influence of the internal region and the functions $R^{(\mathrm{N}, \mathrm{D})}(k) \equiv-2 \tan \delta^{(\mathrm{N}, \mathrm{D})}(k)$ by

$$
\begin{gathered}
R^{(\mathrm{N})}(k)=2 k G_{\text {int }}^{(\mathrm{N})}(a, a)=2 k \sum_{n} \frac{u_{n}^{0}(a) u_{n}^{0}(a)}{k^{2}-\varepsilon_{n}^{(\mathrm{N})}}, \\
R^{(\mathrm{D})}(k)=\frac{2}{k} G_{\mathrm{int}}^{(\mathrm{D})^{\prime \prime}}\left(a_{-}, a\right)=\frac{2}{k} \sum_{n} \frac{v_{n}^{0 \prime}\left(a_{-}\right) v_{n}^{0 \prime}(a)}{k^{2}-\varepsilon_{n}^{(\mathrm{D})}} .
\end{gathered}
$$

The spectral representations of the Green's function are used here in the last steps. This shows that the energy levels of the corresponding internal problems are real poles of the $R$ functions in the complex energy plane. The formulas obtained are in close analogy with the representations found in [17] in a different way. They are quite similar to those appearing in the $R$-matrix theory [4, 28]. We should, however, stress that functions $R$ defined in our manner differ from the standard Wigner's $R$ function by trivial factors like $-2 k$ or $-2 / k$ which we for the sake of convenience include in the definition.
A certain caution is needed while taking the limit $r, r^{\prime} \rightarrow a$ in Eq. (18b) since the derivative of the Green's function

$$
\begin{aligned}
G_{\text {int }}^{(\mathrm{D})}\left(r, r^{\prime}\right) & =\theta\left(r^{\prime}-r\right) \chi_{1}^{0}(r) \chi_{2}^{0}\left(r^{\prime}\right)+\theta\left(r-r^{\prime}\right) \chi_{1}^{0}\left(r^{\prime}\right) \chi_{2}^{0}(r) \\
& =\sum_{n} \frac{v_{n}^{0}(r) v_{n}^{0}\left(r^{\prime}\right)}{k^{2}-\varepsilon_{n}^{(\mathrm{D})}}
\end{aligned}
$$

has a discontinuity when $r=r^{\prime}$. The symbols $\chi_{1}^{0}(r)$ and $\chi_{2}^{0}(r)$ stand for solutions of the internal problem with Dirichlet BCs only at the points $r=0$ or $r=a$, respectively. It is readily seen that the mixed second partial derivative contains a singular contribution

$$
\left[\chi_{1}^{0^{\prime}}(r) \chi_{2}^{0}(r)-\chi_{1}^{0}(r) \chi_{2}^{0^{\prime}}(r)\right] \delta\left(r-r^{\prime}\right)=W \delta\left(r-r^{\prime}\right)
$$

$W=-1$ being the Wronskian. This singularity at $r=$ $r^{\prime}=a$ must be excluded and the second derivative must be understood as

$$
G_{\mathrm{int}}^{(\mathrm{D})}\left(a_{-}, a\right)=\chi_{1}^{0^{\prime}}(a) \chi_{2}^{0^{\prime}}(a) .
$$

It immediately follows from this remark that the spectral sum $\sum_{n} v_{n}^{0^{\prime}}(r) v_{n}^{0^{\prime}}\left(r^{\prime}\right) /\left(k^{2}-\varepsilon_{n}^{(\mathrm{D})}\right)$ diverges when $r=r^{\prime}$. Indeed, convergence of this sum depends on the contributions of the very high levels, $n \rightarrow \infty$. For such an excitation, we can neglect the potential $U(r)$ whereupon the solution of the internal problem reduces simply to $v_{n}^{0}(r)=\sqrt{2 / a} \sin (n \pi r / a)$. The contribution $\delta\left(r-r^{\prime}\right)$ to be dropped appears due to the factor $\varepsilon_{n}^{(\mathrm{D})}=(\pi n / a)^{2}$ which arises in the numerator after the double differentiation has been done. It is easy to see, that just the contribution of this kind mainly caused numerical problems in [17]. For $r \neq r^{\prime}$, the sum converges, although slowly, to the finite limit (21) because of oscillations 29].

It would be a mistake to rely upon the spectral representations in (18), and interpret the eigenvalues $\varepsilon_{n}$ as energies of resonance states and the phases $\delta_{0}^{(\mathrm{D})}(k)=k a$ or $\delta_{0}^{(\mathrm{N})}(k)=k a+\pi / 2$ as smooth phases of the background scattering. Indeed, the levels $\varepsilon_{n}$ depend on what sort of boundary conditions have been used. More than that, many other forms of $\mathrm{BC}$ are equally permissible (see, for example, the fundamental review [28]). It can be shown that the most general possible $\mathrm{BC}$,

$$
u^{0^{\prime}}(a)+\beta_{\mathrm{int}} u^{0}(a)=0, \quad \phi^{0^{\prime}}(a)+\beta_{\mathrm{ext}} \phi^{0}(a)=0,
$$

involve two arbitrary parameters $\beta$ (here we include formally $\beta= \pm \infty$ for the Dirichlet BCs). Returning to Eq. (17), we see that, due to the relation $S_{0}^{(\mathrm{N})}(k)=$ $-S_{0}^{(\mathrm{D})}(k)$, the levels $\varepsilon_{n}^{(\mathrm{D})}$ are zeros of the function $R^{(\mathrm{N})}(k)$ and, similarly, the levels $\varepsilon_{n}^{(\mathrm{N})}$ are zeros of $R^{(\mathrm{D})}(k)$. In fact, the two phases are connected as

$$
\tan \delta^{(\mathrm{D})}(k)=-\cot \delta^{(\mathrm{N})}(k)=\tan \left[\delta^{(\mathrm{N})}(k)-\pi / 2\right] .
$$

The shift $\pi / 2$ just compensates the similar shift of $\delta_{0}^{(\mathrm{N})}(k)$ and the total scattering phase $\delta(k)=\delta^{(\mathrm{N})}(k)+\delta_{0}^{(\mathrm{D})}(k)=$ 
$\delta^{(\mathrm{D})}(k)+\delta_{0}^{(\mathrm{N})}(k)$ does not depend on the type of $\mathrm{BC}$ used. In particular, the positions of the poles of the function $S(k)$ in the complex $k$ plane, which are found from the equation

$$
1-i \tan \delta^{(\mathrm{D})}(k)=0=1-i \tan \delta^{(\mathrm{N})}(k),
$$

are BC independent because of the relation (23). This is in agreement with the fact that both factors $S_{0}^{(\mathrm{D}, \mathrm{N})}$ are entire functions in $k$ plane.

The factorized form of the residues of poles in (18) allows one to represent the function $S(k)$ in a different but fully equivalent form as

$$
S(k)=\left(1-i A^{T} \frac{1}{k^{2}-\mathcal{H}} A\right) S_{0}(k),
$$

where the non-Hermitian symmetric operator $\mathcal{H}$ is defined by

$$
\mathcal{H}(k)=\varepsilon-\frac{i}{2} A A^{T} .
$$

Here $\varepsilon$ is a diagonal matrix of the eigenvalues of the corresponding internal problems and the column vector $A(k)$ of coupling amplitudes, which originate from the off-diagonal element $V$ in Eq. (44), has the components

$$
A_{n}^{(\mathrm{N})}(k)=\sqrt{2 k} u_{n}^{0}(a) \quad \text { or } \quad A_{n}^{(\mathrm{D})}(k)=\sqrt{\frac{2}{k}} v_{n}^{0^{\prime}}(a)
$$

in the cases of Neumann or Dirichlet BC, respectively. A proof of equivalence to the expressions (17) immediately comes from the following relation between resolvents (see, for example, $[9]$ ):

$$
\frac{1}{k^{2}-\mathcal{H}}=G_{\mathrm{int}}-\frac{i}{2} G_{\mathrm{int}} A \frac{1}{1+\frac{i}{2} R} A^{T} G_{\mathrm{int}} .
$$

It follows from Eq. (25) that the poles of the $S$ function can also be found from the secular equation

$$
\operatorname{det}\left[z^{2}-\mathcal{H}(z)\right]=0
$$

where $z$ is a point in the complex $k$ plane. In the position representation this is equivalent to the spectral problem with a complex (outgoing-wave) boundary condition

$$
\begin{aligned}
\left(-\frac{d^{2}}{d r^{2}}+U(r)\right) \psi(r) & =z^{2} \psi(r), \\
\psi^{\prime}(a)-i z \psi(a) & =0 .
\end{aligned}
$$

This clearly demonstrates again the independence of the choice of BCs for disconnected internal and external motions.

Actually, the form of the operator (26) is provocative. One is tempted to interpret this operator as an effective Hamiltonian whose $k$-dependent eigenvalues define complex energies of metastable resonance states formed by the potential $U(r)$. However, this interpretation is, generally, wrong. To make our points clearer, let us start with the following simple remark. Let the potential vanish identically everywhere, $U(r) \equiv 0$, so that the $S$ function $S(k) \equiv 1$. Nevertheless, neither the operator $\mathcal{H}$ nor the function

$$
\tilde{S}(k)=1-i A^{T} \frac{1}{k^{2}-\mathcal{H}} A
$$

are trivial in themselves. For example, in the case of Neumann BCs matrix elements of $\mathcal{H}$ are

$$
\mathcal{H}_{m n}^{(\mathrm{N})}=\frac{1}{a^{2}}\left[\left(m+\frac{1}{2}\right)^{2} \pi^{2} \delta_{m n}-i k a(-1)^{(m+n)}\right] .
$$

Any truncated finite-size $N \times N$ matrix obtained from Eq. (32) gives, when substituted in Eq. (29), $N$ pairs of complex roots. However, the complex poles of the resolvent $\left[z^{2}-\mathcal{H}_{\text {trunc }}(z)\right]^{-1}$ of the truncated matrix have nothing to do with the poles of the genuine $S$ function. The truncation procedure is not stable when $N \rightarrow \infty$. The difficulties become even worse in the case of Dirichlet BCs when the imaginary part also grows,

$$
\mathcal{H}_{m n}^{(\mathrm{D})}=\frac{1}{a^{2}}\left[m^{2} \pi^{2} \delta_{m n}-i \pi^{2} \frac{m n}{k a}(-1)^{(m+n)}\right] .
$$

In fact, all poles found go to infinity in the limit $N \rightarrow$ $\infty$. Indeed, the true poles, because of the identity $\operatorname{det}\left[z^{2}-\mathcal{H}(z)\right]=\operatorname{det}\left(z^{2}-H_{\text {int }}\right)\left[1+\frac{i}{2} R(z)\right]$, must satisfy the equation

$$
1+\frac{i}{2} R(z)=0,
$$

which is equivalent to (24). For example, in the case of Neumann BC this equation runs as $1+i z G_{\text {int }}^{(\mathrm{N})}(a, a)=0$. In particular, when the potential $U(r)$ vanishes identically, the Green's function in the $z$ plane is equal to $G_{\text {int }}^{(\mathrm{N})}(a, a)=-(1 / z) \tan z a$, Eq. (34) looks as $1-$ $i \tan z a=0$ and, therefore, has no roots in any finite domain of this plane. This implies, in turn, that Eq. (30) has no non-trivial solutions. In fact, the function (31) is simply equal to $\tilde{S}_{\text {free }}(k)=e^{2 i k a}$ in this case and compensates exactly the phase shift due to the fictitious reflection at the separation point $a$.

The remark above is of quite a general nature. The radius of separation can be chosen arbitrarily. For the sake of simplicity, we suggest only that $a$ is larger than the finite radius of the potential. The actual wave function satisfies at this point the conditions

$$
\phi(a)=u(a), \quad \phi^{\prime}(a)=u^{\prime}(a) .
$$

Of course, none of these quantities is known before the problem has been solved. Any boundary condition used above generates a complete basis in the Hilbert space, in which the actual wave functions can be expanded. All such bases are formally equivalent. But this does not mean that all of them are equally adequate from the physical point of view. In particular, the more the basis eigenvectors and their derivatives differ at the point $a$ 
from the real values (35) the more slowly the corresponding expansion converges near this point.

A forced BC creates a false reflection at this point, which is described by the factor $S_{0}(k)$, whose phase should be fully compensated by the similar part of the total phase of the function $\tilde{S}(k)$. Both factors separately depend on $a$ although the complete function $S(k)$ does not. The main role in this compensation is played by the matrix elements $\mathcal{H}_{m n}$ with large $m, n$ when the influence of the potential $U$ becomes negligible, and we return to the situation described in the previous paragraph.

However, the choice of the separation radius and $\mathrm{BC}$ influences the positions of the poles (as well as the residues) of the function $R$ and, consequently, the explicit form of the matrix $\mathcal{H}_{m n}$. This influence is stronger the less adequate the choice made of $\mathrm{BC}$ and $a$. In general, the parameters of the $R$ function can carry rather poor information about the actual complex poles. For this reason, diagonalization of a truncated matrix $\mathcal{H}_{m n}$ (which is necessary in any numerical computation) can lead in the case of a poor choice to strong disagreement with the characteristics of the actual poles of the function $S$. The explicit dependence of the matrix elements $\mathcal{H}_{m n}$ on the wave number $k$ causes additional problems (see next section). To extract the physically relevant effective Hamiltonian from the formal operator $\mathcal{H}$, additional physical considerations must be engaged. For example, one may expect from the physical point of view that the most relevant choice of the separation point $a$ would be a distance matching an outer potential barrier which is strong enough to make immediate reflection at this point quite probable.

To explore in more detail the questions briefly discussed above, we will apply in the next section the formal technique sketched here to the problem of scattering by a finite periodic set of $\delta$ barriers, which can be solved exactly.

\section{OPEN KRONIG-PENNEY MODEL}

\section{A. Exact solution}

We will consider below $s$-wave scattering by a periodically disposed chain of $L \delta$ barriers,

$$
U(r)=\sum_{l=1}^{L} \kappa_{l} \delta(r-l) .
$$

To ensure formation of long-lived resonance states, at least some of the strength constants $\kappa_{l}$ should be positive. The distance $r$ is measured in units of the size of the well formed by two neighboring barriers. Below, we derive the effective Hamiltonian starting directly from the Schrödinger equation.

Because of the local character of the barriers, it is most convenient to start from, instead of the Schrödinger equa- tion, the equivalent integral equation

$$
\begin{aligned}
\chi(r) & =\sin (k r)+\int_{0}^{\infty} d r^{\prime} G_{+}^{0}\left(r, r^{\prime}\right) U\left(r^{\prime}\right) \chi\left(r^{\prime}\right) \\
& =\sin (k r)+\sum_{l=1}^{L} \kappa_{l} G_{+}^{0}(r, l) \chi(l) .
\end{aligned}
$$

Here the symbol $G_{+}^{0}\left(r, r^{\prime}\right)=-(1 / k) g^{0}\left(r, r^{\prime}\right)$ with

$$
g^{0}\left(r, r^{\prime}\right)=\theta\left(r^{\prime}-r\right) \sin (k r) e^{i k r^{\prime}}+\theta\left(r-r^{\prime}\right) \sin \left(k r^{\prime}\right) e^{i k r}
$$

stands for the Green's function of the free radial motion which has outgoing-wave asymptotic. From the second line in (37) we find immediately $S(k)=1-i T(k)$ where the scattering amplitude is given by

$$
T(k)=\frac{2}{k} \sum_{l=1}^{L} \kappa_{l} \sin (k l) \chi(l)=\frac{2}{k} s^{\mathrm{T}} \kappa \chi .
$$

In the second equality we have used matrix notation, with $s$ and $\chi$ being $L$-dimensional vectors with the components $s_{l} \equiv s_{l}(k) \equiv \sin (k l)$ and $\chi_{l} \equiv \chi(l)(l=1,2, \ldots, L)$, respectively, when $\kappa=\operatorname{diag}\left\{\kappa_{1}, \kappa_{2}, \ldots, \kappa_{\mathrm{L}}\right\}$.

According to Eq. (37), the $L$-dimensional vector $\chi$ satisfies the equation $\chi=s+G^{0} \kappa \chi$, where $G^{0}=-(1 / k) g^{0}$ and $g^{0}$ is a symmetric non-Hermitian matrix with the matrix elements

$$
g_{l l^{\prime}}^{0}= \begin{cases}\sin (k l) e^{i k l^{\prime}} & \text { if } \quad l \leq l^{\prime}, \\ \sin \left(k l^{\prime}\right) e^{i k l} & \text { if } \quad l>l^{\prime} .\end{cases}
$$

Thus, we obtain finally

$$
\begin{aligned}
S(k) & =e^{2 i \delta(k)}=1-i s^{\mathrm{T}} \mathcal{G}(k) s=\frac{1-\frac{i}{2} K(k)}{1+\frac{i}{2} K(k)}, \\
K(k) & =-2 \tan \delta(k)=s^{\mathrm{T}} G(k) s,
\end{aligned}
$$

where we have used the factorized form $\Im \mathrm{m} g^{0}=s s^{\mathrm{T}}$ of the anti-Hermitian part of the matrix $g^{0}$ to pass from the first to the second equality. The $L \times L$ matrix propagators

$$
\mathcal{G}(k)=\frac{2}{k \lambda+g^{0}} \quad \text { and } \quad G(k)=\frac{2}{k \lambda+\Re e g^{0}}
$$

are connected with one another by a relation similar to (28). The diagonal matrix $\lambda=\kappa^{-1}$ characterizes the penetrabilities of the barriers [30]. The poles of the $S$ function are defined by the equation

$$
\operatorname{det}\left[z \lambda+g^{0}(z)\right]=0=1+\frac{i}{2} K(z) .
$$

All the matrix elements (39) are entire functions in the complex $z$ plane. The same is valid for the determinant in (42). Therefore, one can show that this equation has an infinite number of isolated complex roots. Further, its roots because of the relation $\left[g_{l l^{\prime}}^{0}(z)\right]^{*}=-g_{l l^{\prime}}^{0}\left(-z^{*}\right)$ come in pairs $z_{n}$ and $z_{-n} \equiv-z_{n}^{*}, n=1,2, \ldots$, symmetrically with respect to the imaginary axis, or lie on the latter, $z_{q}=$ 
$i y_{q}$. All poles of the first type are situated in the lower part of the complex plane. Poles on the positive half of the imaginary axis, $y_{q}>0$, correspond to bound states and can appear only if some number of the constants $\kappa_{l}$ are negative. Those which are situated on the negative part, $y_{q}<0$, correspond to the so-called virtual levels. The total number of purely imaginary poles is finite for any finite $L$.

After all poles have been found, the function $S(k)$ can be presented in the form

$$
S(k)=\prod_{n=1}^{\infty} \frac{\left(k+z_{n}\right)\left(k-z_{n}^{*}\right)}{\left(k-z_{n}\right)\left(k+z_{n}^{*}\right)} \prod_{q} \frac{k+i y_{q}}{k-i y_{q}}
$$

where we took into account that $S(k=\infty)=1$, since $\delta$ barriers become transparent for a particle with asymptotically large energy. These expressions are in agreement with the general theory [31, 32], which is valid for any potential with a finite radius.

Each factor in (43) is singly unitary. In particular, for a pair $z_{ \pm n}$ of conjugate roots we have

$$
\begin{aligned}
S_{n}(k) & \equiv \frac{\left(k+z_{n}\right)\left(k-z_{n}^{*}\right)}{\left(k-z_{n}\right)\left(k+z_{n}^{*}\right)} \\
& =\frac{k^{2}-\left|z_{n}\right|^{2}+2 i k \Im \mathrm{m} z_{n}}{k^{2}-\left|z_{n}\right|^{2}-2 i k \Im \mathrm{m} z_{n}} \equiv e^{2 i \delta_{n}(k)} .
\end{aligned}
$$

Since $\Im \mathrm{m} z_{n}<0$, the phase $\delta_{n}(k)$ increases when the energy $E=k^{2}$ grows and passes the value $\pi / 2$ at the point $E=\left|z_{n}\right|^{2} \equiv E_{n}$. The typical energy interval $\Delta E$ of the main gain of the phase is estimated by the quantity $2 k\left|\Im \mathrm{m} z_{n}\right|$. If this interval is small enough and the latter quantity does not vary appreciably within it, the total gain is close to $\pi$ and the factor $S_{n}$ receives the standard Breit-Wigner resonance form

$$
S_{n}^{(r e s)}(k)=\frac{E-E_{n}-\frac{i}{2} \Gamma_{n}}{E-E_{n}+\frac{i}{2} \Gamma_{n}} \equiv \frac{E-\mathcal{E}_{n}^{*}}{E-\mathcal{E}_{n}},
$$

with the energy $E_{n} \equiv \Re e \mathcal{E}_{n}$ and width $\Gamma_{n} \equiv-2 \Im m \mathcal{E}_{n}$ of the resonance defined as follows

$$
E_{n}=\left|z_{n}\right|^{2}, \quad \Gamma_{n}=4\left|\Re \mathrm{e} z_{n} \Im \mathrm{m} z_{n}\right| .
$$

The $k$-dependence is neglected in the definition of the width and substitution $k=\sqrt{E} \approx\left|\Re \mathrm{e} z_{n}\right|$ has been made. Such a substitution is well justified when the scattering energy is large enough, but becomes improper near $E=$ 0 . Due to the "threshold" $\sqrt{E}$ dependence of the widths, some specific behavior takes place when in the proximity of this point there exists a number of bound and decaying states [23, 33, 34].

We conclude that the scattering amplitude $T(k)=$ $i[S(k)-1]$ is a meromorphic function in the $z$ plane and can therefore also be written down as an infinite sum of the pole contributions

$$
T(k)=\sum_{n=-\infty}^{\infty} \frac{\varrho_{n}}{k-z_{n}}+\sum_{q} \frac{\varrho_{q}}{k-i y_{q}},
$$

where the residues $\varrho_{n, q}$ can easily be found from Eq. (43).

Another representation of the scattering amplitude sometimes considered in the literature ensues from diagonalization of the (finite in our case) matrix $\mathcal{G}(k)$ at a given fixed real $k$. Since this matrix is complex symmetric, such a diagonalization is performed by a complex orthogonal transformation defined together with the complex eigenvalue matrix $\Lambda(k)=\operatorname{diag}\left\{\Lambda_{1}(k), \Lambda_{2}(k), \ldots, \Lambda_{L}(k)\right\}$ from the equation

$$
\left[k \lambda+g^{0}(k)\right] \Psi(k)=\Psi(k) \Lambda(k) .
$$

The scattering amplitude reduces then to a finite sum

$$
T(k)=2 \sum_{l=1}^{L} \frac{\left[s^{\mathrm{T}}(k) \Psi^{(l)}(k)\right]^{2}}{\Lambda_{l}(k)},
$$

where $\Psi^{(l)}(k)$ is the $l$ th eigenvector. However, such a representation is of limited practical use inasmuch as the terms of the sum are, generally, extremely complicated. There is no simple interpretation of a given term and all of them can give comparable contributions. The compatibility of the two representations (47) and (49) is very indirect. A particular term of the second one cannot be uniquely continued from the real axis at an arbitrary point $z$ in the complex plane because both $\Psi^{(l)}(z)$ and $\Lambda_{l}(z)$ are multivalued functions in this plane. The same is, of course, also true for the opposite direction. Therefore, even if a root $z_{j}^{l}$ of the equation $\Lambda_{l}(z)=0$ is found and near this point the pole approximation

$$
T_{j}^{l}(z) \approx \frac{2\left[s^{\mathrm{T}}\left(z_{j}^{l}\right) \Psi^{(l)}\left(z_{j}^{l}\right)\right]^{2} / \Lambda_{l}^{\prime}\left(z_{j}^{l}\right)}{z-z_{j}^{l}}
$$

is valid, this expression cannot, generally speaking, be continued on the real axis by simply substituting $z \rightarrow k$, since the power expansion $\Lambda_{l}(z)=\Lambda_{l}\left(z_{j}^{l}\right)+\Lambda_{l}^{\prime}\left(z_{j}^{l}\right)(z-$ $\left.z_{j}^{l}\right)+\ldots$ has a finite radius of convergence. Further, for any fixed $l$ a set of roots exists depending on the branch of the function $\Lambda_{l}(z)$ considered. There is no one-to-one correspondence between the set of roots $z_{j}^{l}$ and the manifold of actual poles $z_{n, q}$ that appears in the expansion (47). Many of the roots $z_{j}^{l}$ are false and their contributions must finally cancel out. Therefore, diagonalization of the propagator with non-trivial energy dependence is not as a rule useful; rather it can lead to misleading conclusions.

We compare below the closed expressions Eq. (40) found with the formalism described in the previous section. In correspondence with our remark at the end of Sec. III, we fix the separation point $a$ by superposing it on the position of the outer barrier, $a=L$. The appearance of an additional $\delta$ function changes the corresponding boundary conditions. In particular, if the $\delta$-terms are ascribed to the internal region, Neumann BC (7) for the internal problem is replaced by

$$
\kappa_{\mathrm{L}} u^{0}(a)+u^{0^{\prime}}(a)=0=u^{0}(a)+\lambda_{\mathrm{L}} u^{0^{\prime}}(a),
$$


whereas attributing such terms to the external domain yields the following change of the $\mathrm{BC}$ of the external problem:

$$
\kappa_{\mathrm{L}} \phi^{0}(a)-\phi^{0^{\prime}}(a)=0=\phi^{0}(a)-\lambda_{\mathrm{L}} \phi^{0^{\prime}}(a) .
$$

We stress that neither of these conditions coincides with the boundary conditions

$$
\begin{aligned}
& \chi\left(a_{-}\right)=\chi\left(a_{+}\right) \equiv \chi(a), \\
& \chi^{\prime}\left(a_{+}\right)-\chi^{\prime}\left(a_{-}\right)=\kappa_{\mathrm{L}} \chi(a)
\end{aligned}
$$

satisfied by the exact wave function $\chi(r)$.

In the case (52), the immediate reflection at the point $a$ is described instead of $S_{0}^{(\mathrm{N})}$ by

$$
S_{0}(k)=S_{0}^{(\mathrm{N})} \frac{k-i \kappa_{\mathrm{L}}}{k+i \kappa_{\mathrm{L}}}=\frac{1+i \lambda_{\mathrm{L}} k}{1-i \lambda_{\mathrm{L}} k} S_{0}^{(\mathrm{D})} .
$$

(Recall that $S_{0}^{(\mathrm{D})}(k)=e^{-2 i k a} \equiv e^{-2 i k L}$.) The additional factor is due to the influence of the barrier outside the radius of separation $a$. This factor has a pole $k=-i \kappa_{\mathrm{L}}$ on the negative part of the imaginary axis, which formally corresponds to a virtual Wigner level. Actually, such a pole does not exist in the exact solution and disappears due to cancellation with the contribution of the internal region [see Eq. (95) below].

\section{B. Internal problem with Neumann BC}

The exact $S$ function in the case of the BC (51) reads as $S(k)=\tilde{S}(k) S_{0}^{(\mathrm{D})}(k)$, where

$$
\tilde{S}(k)=\frac{1-\frac{i}{2} R}{1+\frac{i}{2} R}=1-i \tilde{T}(k),
$$

with

$$
\begin{aligned}
R(k) & =\tilde{A}^{T} \frac{1}{k^{2}-H_{\mathrm{int}}^{(\mathrm{N})}} \tilde{A}=2 k G_{\mathrm{int}}^{(\mathrm{N})}(a, a) \\
& =2 k \lambda_{\mathrm{L}}^{2} \sum_{n=1}^{\infty} \frac{u_{n}^{0 \prime}(a) u_{n}^{0 \prime}(a)}{k^{2}-\varepsilon_{n}^{(\mathrm{N})}}
\end{aligned}
$$

and

$$
\tilde{T}(k)=\tilde{A}^{T} \frac{1}{k^{2}-\mathcal{H}^{(\mathrm{N})}} \tilde{A} .
$$

The coupling amplitudes are equal to

$$
\tilde{A}_{n}(k)=\sqrt{2 k} u_{n}^{0}(a)=-\lambda_{\mathrm{L}} \sqrt{2 k} u_{n}^{0^{\prime}}(a) .
$$

Finally, the matrix elements of the operator $\mathcal{H}^{(\mathrm{N})}$ [see Eq. (26)] appear as

$$
\begin{aligned}
\mathcal{H}_{m n}^{(\mathrm{N})} & =\varepsilon_{m}^{(\mathrm{N})} \delta_{m n}-i k u_{m}^{0}(a) u_{n}^{0}(a) \\
& =\varepsilon_{m}^{(\mathrm{N})} \delta_{m n}-i \lambda_{\mathrm{L}}^{2} k u_{m}^{0}{ }^{\prime}(a) u_{n}^{0^{\prime}}(a),
\end{aligned}
$$

with the levels $\varepsilon_{n}^{(\mathrm{N})}$ being the eigenvalues of the internal problem with the BC (51). For the sake of simplicity, we use the same superscript $\mathrm{N}$ as before.

In spite of the seemingly similar general structure of the expressions (40), (41) on one hand side and (55) - (57) on the other, they are, in essence, quite different. The most important distinction shows itself in the dimension of the vectors and matrices which is finite and coincides with the number of barriers in the first case and infinite in the second. In addition, the factor $S_{0}(k)$ of the immediate reflection does not appear explicitly in Eq. (40). We will analyze below a couple of simple special cases before drawing general conclusions.

\section{One $\delta$ barrier}

In this case we find immediately from Eqs. (40) $(a=$ $L=1$ and we drop the subscript $L$ in the strength of the barrier)

$$
\begin{aligned}
S(k) & =\frac{\sin k e^{-i k}+\lambda k}{\sin k e^{i k}+\lambda k}, \\
K(k) & =\frac{2 \sin ^{2} k}{\sin k \cos k+\lambda k},
\end{aligned}
$$

whereas

$$
\begin{aligned}
\tilde{S}(k) & =\frac{\sin k+\lambda k e^{i k}}{\sin k+\lambda k e^{-i k}}, \\
R(k) & =-2 \lambda \frac{k \sin k}{\sin k+\lambda k \cos k} .
\end{aligned}
$$

In the Eqs. (61) the phase $\delta^{(\mathrm{D})}(k)=-k$ of the immediate reflection at the point $a$ is extracted. The two functions $K(k)$ and $R(k)$ are related to each other by

$$
K(k)=\frac{2 \tan k+R(k)}{1-\frac{1}{2} \tan k R(k)},
$$

The positions of the poles of the function $S$ coincide with the complex roots of the equation

$$
e^{2 i z}+2 i \lambda z-1=0 .
$$

In the right half of the $z$ plane they can be searched for in the form $z_{n}=n \pi+\zeta_{n}$, where any $\zeta_{n}$ is restricted to the strip $\left|\Re \mathrm{e} \zeta_{n}\right| \leq \pi / 2$ and satisfies the equation

$$
\zeta_{n}+\frac{i}{2} \ln \left[1-2 i \lambda\left(n \pi+\zeta_{n}\right)\right]=0 .
$$

There also exists the trivial root $z=0$ but this root fully cancels out. Even for not very large $n$ one can omit $\zeta_{n}$ in the logarithmic term. This yields the approximate solution

$$
\zeta_{n} \cong-\frac{i}{2} \ln (1-2 i \lambda n \pi)
$$


which is valid with good accuracy for almost all poles, being asymptotically exact when $n \rightarrow \infty$.

However, by no means all of the complex roots $z_{n}$ correspond to resonances. For a pole to correspond to a long-lived resonance state, the following two additional conditions also must be satisfied: i) $\Re \mathrm{e} z_{n}$ should be a real pole of the function $K(k)$ or at least should be close to such a pole; ii) the corresponding residue should be small enough for the scattering phase $\delta(k)$ to increase near this point by $\pi$ in an interval $\Delta k \ll 1$. The validity of these conditions depends on the value of the parameter $\lambda$. Indeed, the poles of $K(k)$ are found from the equation

$$
\sin k \cos k+\lambda k=0 .
$$

There exists only a finite number of real roots $\pm k_{j}$, $j=1,2, \ldots, j_{\max }$ of this equation. They satisfy the requirement $2 \lambda k_{j}<1$ or, equivalently, $k_{j}<\kappa / 2$. (The trivial solution $k=0$ should be dismissed.) All other roots lie in the complex plane and the phase does not reach its maximal value although can change rather fast if a pole of $K$ is still close to the real axis. It is clear that they move away from this axis when $|z|$ grows. In particular, no real solutions exist if $\lambda>\lambda_{0} \approx 0.2\left(\kappa<\kappa_{0} \approx 5\right)$. The scattering phase $\delta(k)<\pi / 2$ in this case and smoothly depends on $k$. The barrier is too weak to form a long-lived resonance state. When $\kappa|\sin k / k|<1$ the scattering phase can be calculated as $\delta(k) \approx-2 \kappa \sin ^{2} k / k+\cdots$ in the framework of perturbation theory.

There are two kinds of real roots of Eq. (65) when $\lambda$ is appreciably less than $\lambda_{0}$. The most interesting case $\lambda k_{j} \ll 1$, although $j \gg 1$, can easily be considered analytically. The first set consists of the roots $k_{n}$ which are close to the points $n \pi: k_{n} \approx\left(1-\lambda+\lambda^{2}\right) n \pi,(n \pi<1 / \lambda)$. Near such a pole the $K$ function manifests typical resonance behavior

$$
K(k) \approx \frac{2(\lambda n \pi)^{2}}{k-k_{n}} .
$$

Strictly speaking, a similar contribution of the symmetric root $k_{-n}=-k_{n}$, which corresponds to the same energy $\varepsilon_{n}=k_{n}^{2}$, should be added, so we arrive near the $n$th resonance at

$$
K_{n}(E)=\frac{(2 \lambda)^{2}(n \pi)^{3}}{E-\varepsilon_{n}} .
$$

Each neighboring pair of resonance roots is separated by a root of the second set: $k_{m} \approx\left[1+(\lambda m \pi)^{-1}\right]\left(m+\frac{1}{2}\right) \pi$, in a vicinity of which

$$
K(k) \approx \frac{2}{k-k_{m}} .
$$

The residues are large in this case and such terms contribute into the smooth background part of the total scattering phase. Indeed, the (dimensionless) time delay $\tau(k)=d \delta(k) / d k=\tau_{w} /\left|\tau_{0}\right|$, which measures the Wigner
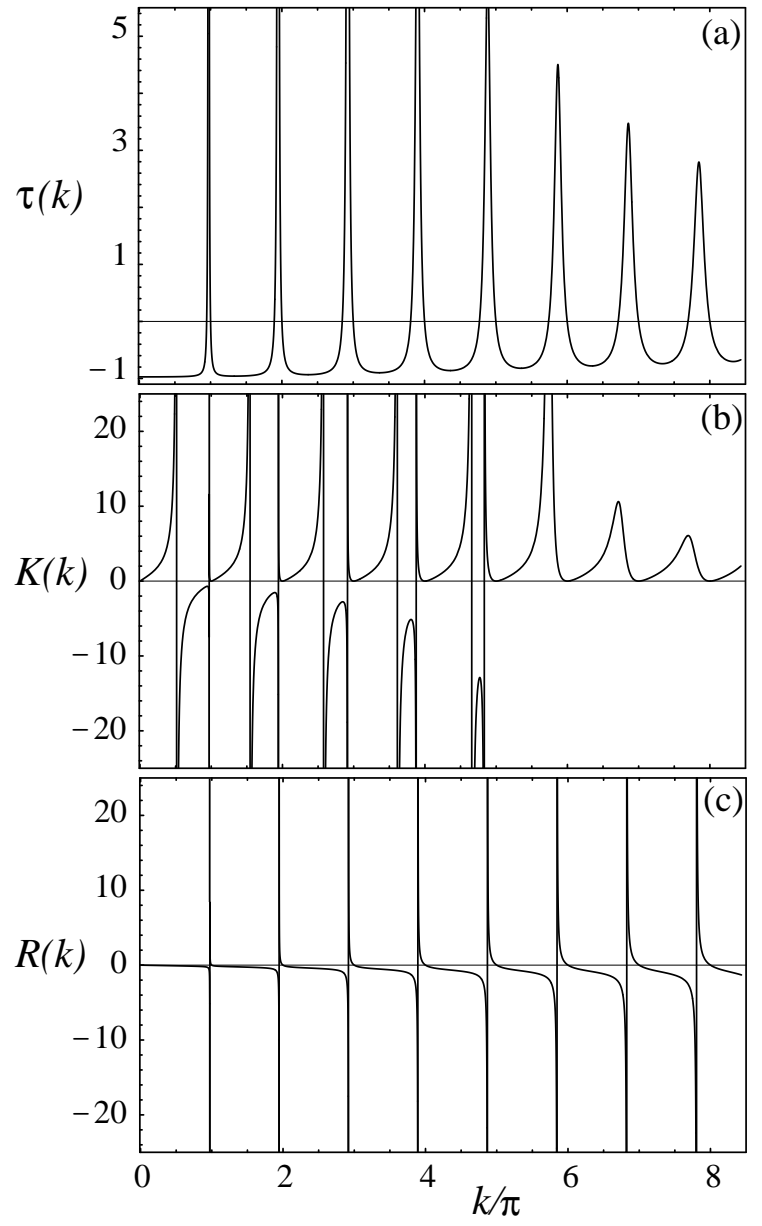

FIG. 1: One $\delta$ barrier model for $\lambda=1 / 35$. (a) The time delay $\tau(k)=d \delta(k) / d k$, (b) $K$ function and (c) $R$ function. Only a finite number $n_{\text {res }} \approx[1 / 2 \pi \lambda](=5)$ of poles located near the points $\pi n, n=1, \ldots, n_{\text {res }}$, correspond to long-lived resonance states; the others refer to the smooth phase of reflection at the point $a(=1)$.

time delay $\tau_{w}=2 d \delta / d E$ in units of the (negative) time delay $\tau_{0}=-2 a /(d E / d k)$ because of the immediate reflection at the point $a(=1)$, is large near the points $k_{n}$ but small when $k \approx k_{m}$. Poles of the two different kinds alternate and resonant and smooth contributions are mixed. Figure 1 clearly demonstrates all the features described above.

Let us on the other hand consider the properties of the function $R(k)$ [see Eq. [61)]. Its poles are the roots of the equation

$$
\sin k+\lambda k \cos k=0
$$

instead of Eq. (65). Contrary to the latter, all roots $\pm \tilde{k}_{n}$ of (69) are real. Those of them, that satisfy the inequality $\lambda \tilde{k}_{n} \ll 1$ and, therefore, have numbers $n<n_{\max }$, are equal to $\tilde{k}_{n} \approx(1-\lambda) n \pi$ again and correspond to resonances. The difference from the similar roots of Eq. (65) appears only at higher order corrections in the parameter $\lambda$. For larger $n$, when $\lambda \tilde{k}_{n} \gg 1$, an infinite number 
of roots $\tilde{k}_{n} \approx \pi\left(n+\frac{1}{2}\right)\left[1+\lambda^{-1}(n \pi)^{-2}\right]$ exists, giving a smooth contribution, which combines with the phase $\delta_{0}^{(\mathrm{D})}(k)=-k$ and almost compensates it at large $k$ [see Fig. 1(c)]. Indeed, the $\delta$ barrier becomes almost transparent when the collision energy is large and the total scattering phase can be calculated by applying perturbation theory.

The meromorphic character of the function $R(k)$ allows us to represent this function in the form of the pole expansion

$$
\begin{aligned}
R(k) & =\sum_{n=-\infty}^{\infty} \frac{2}{1+\lambda+\left(\lambda \tilde{k}_{n}\right)^{2}} \frac{\left(\lambda \tilde{k}_{n}\right)^{2}}{k-\tilde{k}_{n}} \\
& =2 k \sum_{n=1}^{\infty} \frac{2}{1+\lambda+\left(\lambda \tilde{k}_{n}\right)^{2}} \frac{\left(\lambda \tilde{k}_{n}\right)^{2}}{k^{2}-\tilde{\varepsilon}_{n}}
\end{aligned}
$$

The symmetry connection $\tilde{k}_{-n}=-\tilde{k}_{n}$ has been taken into account in the second equality.

Returning to the poles of $S(k)$, we can expand the expression (64) in the resonant region $2 \lambda n \pi \ll 1$ with respect to this parameter to calculate the poles

$$
z_{n} \approx\left(1-\lambda+\lambda^{2}\right) n \pi-i(\lambda n \pi)^{2}
$$

and the complex energies of the resonance states

$$
\mathcal{E}_{n} \approx\left(1-2 \lambda+3 \lambda^{2}\right)(n \pi)^{2}-\frac{i}{2}\left[4 \lambda^{2}(n \pi)^{3}\right] .
$$

Note that the widths $\Gamma_{n}=4 \lambda^{2}(n \pi)^{3}$ of the resonances appear only in the second order in the penetrability parameter $\lambda$ when the shifts of their energies are of the first order of magnitude. The resonances are well isolated since the ratio $\Gamma_{n} /\left(E_{n+1}-E_{n}\right)=2 \pi(\lambda n)^{2} \ll 1$. Finally, the remote poles with $n \gg 1 / 2 \pi \lambda$ are given by

$$
z_{n} \approx\left(n-\frac{1}{4}\right) \pi-\frac{i}{2} \ln (2 \lambda n \pi)^{2} .
$$

Now we compare our findings with the results (55)(57) of the general formalism. The normalized solutions of the internal problem with $\mathrm{BC}$ (51) are readily found to be

$$
u_{n}^{0}(r)=\sqrt{\frac{2}{1+\lambda \cos ^{2} k_{n}}} \sin \left(k_{n} r\right)
$$

where $k_{n}$ are the roots of Eq. (69) which follows directly from the $\mathrm{BC}$ (51) this time (we omit the tilde and note that only positive roots $k_{n}, n=1,2, \ldots$, are to be kept). This fact is quite satisfactory and demonstrates the physical relevance of the choice of $\mathrm{BC}$ made. Indeed, for such a BC $u_{n}^{0}(a) \sim \lambda$ in accordance with the exact boundary condition (53). The Dirichlet BC $u_{n}^{0}(a)=0$ would be deficient in this sense. With Eq. (74) taken into account, we find from Eq. (56)

$$
R(k)=2 k \sum_{n=1}^{\infty} \frac{2 \cos ^{2} k_{n}}{1+\lambda \cos ^{2} k_{n}} \frac{\left(\lambda k_{n}\right)^{2}}{k^{2}-\varepsilon_{n}} .
$$

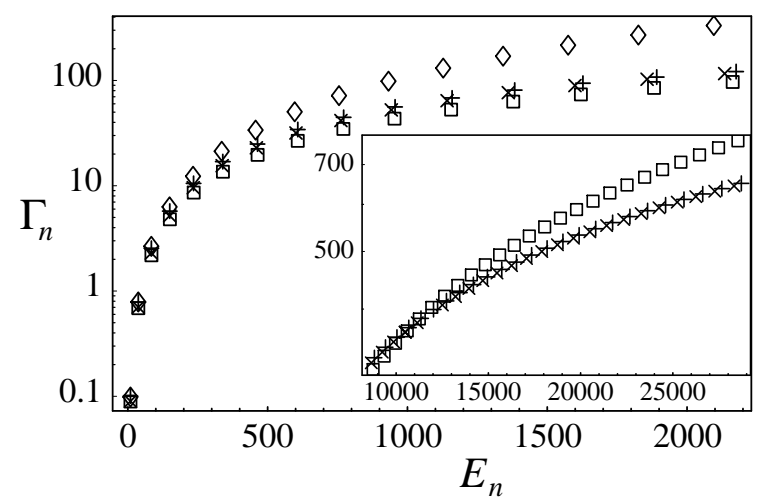

FIG. 2: Widths (in log scale) versus energies for $\lambda=1 / 35$. Exact poles [from Eq. [63), $\square$ ] and approximation [Eq. (72), $\diamond]$, compared to the "diagonal" approximation $\mathcal{H}_{n n}\left(k=k_{n}\right)$ with Neumann [from Eq. (76), $\times$ ] or Dirichlet BC [from Eq. (97), +]. Inset shows the asymptotic region of remote poles, where the diagonal approximation breaks down.

Equivalence to Eq. (70) is seen from the relation $\cos ^{2} k_{n}=\left[1+\left(\lambda k_{n}\right)^{2}\right]^{-1}$, which follows directly from the secular equation (69).

Matrix elements of the operator $\mathcal{H}(59)$ are in our case

$\mathcal{H}_{m n}^{(\mathrm{N})}=k_{n}^{2} \delta_{m n}-i 2 k \frac{\lambda k_{m}}{\sqrt{1+\lambda+\left(\lambda k_{m}\right)^{2}}} \frac{\lambda k_{n}}{\sqrt{1+\lambda+\left(\lambda k_{n}\right)^{2}}}$.

In the resonance domain, $\lambda k_{n} \lesssim 1$, the diagonal matrix element $\mathcal{H}_{n n}^{(\mathrm{N})}\left(k=k_{n}\right)$ approximates well the complex energy $\mathcal{E}_{n}$ of the resonance which lies near the scattering energy $E \approx k_{n}^{2}$. In this region off-diagonal elements influence only corrections of higher orders. At the same time, the diagonal approximation becomes insufficient for the remote poles, breaking down to reproduce exact asymptotic $n \ln n$ behavior of the imaginary parts. Figure 2 illustrates the consideration.

We therefore conclude that the extraction of the phase $\delta_{0}^{(\mathrm{D})}(k)$ of the immediate reflection and utilization of the $R$-function is adequate only in the resonance region where such a reflection is probable. Beyond this region the internal and immediate reflection phase shifts almost compensate each other so that the function $K(k)$ proves to be a more relevant tool.

\section{Resonance domain}

Below, we restrict our attention to the resonance region well above the energy $E=k^{2}=0$. To simplify the investigation further, we suggest assuming the strength constants to be the same $\lambda_{l}=\lambda_{1}, l=2,3, \ldots, L-1$, for all inner barriers. In accordance with the results of the simple consideration presented at the end of Sec. IV C, segregation of the smooth phase of the immediate reflection meets expectations based on physical intuition. 
To perform this segregation, we write first the scattering amplitude $T(k)=s^{\mathrm{T}} \mathcal{G}(k) s$ as a block product

$$
\begin{aligned}
T(k) & =\left(\mathbf{s}^{\mathrm{T}}, s_{\mathrm{L}}\right)\left(\begin{array}{cc}
\hat{\mathcal{G}} & \mathcal{F} \\
\mathcal{F}^{T} & \mathcal{G}_{\mathrm{L}}
\end{array}\right)\left(\begin{array}{c}
\mathbf{s} \\
s_{\mathrm{L}}
\end{array}\right) \\
& =\left[\left(\lambda_{\mathrm{L}} k-s_{\mathrm{L}} e^{i k a}\right) \mathbf{s}^{\mathrm{T}} \frac{1}{\lambda_{1} k \hat{I}+\hat{g}^{0}} \mathbf{s}+s_{\mathrm{N}}^{2}\right] \mathcal{G}_{\mathrm{L}},
\end{aligned}
$$

where the symbols $\hat{\mathcal{G}}$ and $\mathcal{F}$, etc., stand for $(L-1) \times$ $(L-1)$ submatrices and $(L-1)$-dimensional vectors, respectively, and $\hat{g}^{0}$ is the similar upper left block of $g^{0}$. To pass from the first to the second line, the relations

$$
\mathbf{s}^{\mathrm{T}} \hat{\mathcal{G}} \mathbf{s}=-\left(\lambda_{\mathrm{L}} k e^{-i k a}+s_{\mathrm{L}}\right) \mathbf{s}^{\mathrm{T}} \mathcal{F}
$$

and

$$
\mathbf{s}^{\mathrm{T}} \mathcal{F}=\mathcal{F}^{\mathrm{T}} \mathbf{s}=-e^{i k a} \mathbf{s}^{\mathrm{T}} \frac{1}{\lambda_{1} k \hat{I}+\hat{g}^{0}} \mathbf{s}
$$

have been used which follow, together with the expression

$$
\mathcal{G}_{\mathrm{L}}=2\left[\lambda_{\mathrm{L}} k e^{-i k a}+s_{\mathrm{L}}-e^{i k a} \mathbf{s}^{\mathrm{T}} \frac{1}{\lambda_{1} k \hat{I}+\hat{g}^{0}} \mathbf{s}\right]^{-1} e^{-i k a},
$$

from the equation $\left(k \lambda+g^{0}\right) \mathcal{G}(k)=2$. We notice now that the function

$$
T_{\infty}(k)=\mathbf{s}^{\mathrm{T}} \frac{2}{\lambda_{1} k \hat{I}+\hat{g}^{0}} \mathbf{s}
$$

coincides with the amplitude of scattering on the potential (36) with the last barrier being removed: $\kappa_{\mathrm{L}}=0$ or, in other words, $\lambda_{\mathrm{L}}=\infty$. Finally, we obtain after simple transformations $S(k)=\tilde{S}(k) e^{-2 i k L}$, where

$$
\begin{aligned}
\tilde{S}(k) & =\frac{e^{-2 i k L}-\left(1+2 i \lambda_{\mathrm{L}} k\right) S_{\infty}(k)}{\left(1-2 i \lambda_{\mathrm{L}} k\right) e^{-2 i k L}-S_{\infty}(k)} \\
& =\frac{\sin \left(\delta_{\infty}+k a\right)+\lambda_{\mathrm{L}} k e^{i\left(\delta_{\infty}+k a\right)}}{\sin \left(\delta_{\infty}+k a\right)+\lambda_{\mathrm{L}} k e^{-i\left(\delta_{\infty}+k a\right)}} .
\end{aligned}
$$

The poles of the function $\tilde{S}(k)$ [as well as of $S(k)$ ] in the complex $k$ plane are given by zeros of the denominator. It is convenient to introduce in parallel with this function a sequence of functions $\tilde{S}^{(l)}(k) \equiv S^{(l)}(k) e^{2 i l k}$ which describe the scattering on chains of $l$ barriers with the penetrability constant $\lambda_{1}$ and the BC fixed at the radius $a=l$. In particular, $\tilde{S}^{(L)}(k) \equiv \tilde{S}(k)$, $\tilde{S}^{(L-1)}(k) \equiv S_{\infty}(k) e^{2 i(L-1) k}$, and $\tilde{S}^{(0)}(k) \equiv 1$. These functions are related to each other by a recursion

$$
\tilde{S}^{(l)}(k)=\frac{1-\left(1+2 i \lambda_{1} k\right) e^{2 i k} \tilde{S}^{(l-1)}(k)}{1-2 i \lambda_{1} k-e^{2 i k} \tilde{S}^{(l-1)}(k)} .
$$

Using as before the ansatz $z_{m}=m \pi+\zeta_{m}$ in the resonance region $\lambda_{1, \mathrm{~L}}|z| \approx \lambda_{1, \mathrm{~L}}|m| \pi \ll 1$, we arrive at an algebraic equation $\mathcal{P}^{(\mathrm{L})}\left(e^{2 i \zeta_{m}}\right)=0$, with $\mathcal{P}(w)$ being a polynomial of the $L$ th power with respect to the argument $w=e^{2 i \zeta_{m}}$. This equation gives a bunch of $L$ close complex poles of the $S$ function well separated from all the other poles.

The corresponding $R$ function is readily found from Eq. (79) to be

$$
R(k)=-\frac{2}{1 / \lambda_{\mathrm{L}} k+\cot \left(\delta_{\infty}+k a\right)} .
$$

In the limit $\lambda_{\mathrm{L}} \rightarrow 0$ (closed interior) we have $R(k) \equiv 0$ and $\tilde{S}(k) \equiv 1$, so that only the immediate reflection at the point $a$ survives. Obviously, the spectrum of the poles of the function $R(k)$ is determined by the equation

$$
\sin \left(\delta_{\infty}+k a\right)+\lambda_{\mathrm{L}} k \cos \left(\delta_{\infty}+k a\right)=0
$$

which should be compared with Eq.! 69. It is easy to check that the spectrum exactly coincides with that of the wave numbers of the internal problem for the potential $U_{\infty}(r)=\kappa_{1} \sum_{l=1}^{L-1} \delta(r-l)$ with the BC (51). This potential is perfectly transparent on the separation radius $a=L$ where the latter condition is fixed. This directly follows from the expression

$$
u^{0}(r)=\sin (k r)-\frac{1}{2} T_{\infty}(k) e^{i k r}=\frac{i}{2}\left[e^{-i k r}-S_{\infty}(k) e^{i k r}\right]
$$

for the wave function in the region $(L-1)<r \leq a=L$.

\section{E. Two $\delta$ barriers; resonance trapping}

Now we will use the formulaes just found to analyze as an illustrative example resonances in the double-well $\delta$-potential, $L=2$. The equation for the poles of the $S$ function (79) looks in this case like

$$
\begin{gathered}
\left(1+2 i \lambda_{1} z\right) e^{4 i z}-2\left(1-2 i \lambda_{2} z\right) e^{2 i z} \\
+\left(1-2 i \lambda_{1} z\right)\left(1-2 i \lambda_{2} z\right)=0
\end{gathered}
$$

[cf. Eq. (62)]. Again, the ansatz $z_{m}=m \pi+\zeta_{m}$ with $\left|\Re \mathrm{e} \zeta_{m}\right| \leq \pi / 2$ is valid. Supposing also the integer number $m$ to be appreciably large, we arrive for each fixed $m$ at a couple of closed solutions [compare with (64)]

$$
\begin{aligned}
\zeta_{m} \approx & -\frac{i}{2} \ln \left\{1-i \frac{\left(2 \lambda_{1}+\lambda_{2}\right) m \pi}{1+2 i \lambda_{1} m \pi}\right. \\
& \left.\times\left[1 \pm \sqrt{1-\frac{4 \lambda_{1} \lambda_{2}}{\left(2 \lambda_{1}+\lambda_{2}\right)^{2}}\left(1+2 i \lambda_{1} m \pi\right)}\right]\right\} .
\end{aligned}
$$

It is worth noting that within the approximation adopted the sum of the imaginary parts $\Im m\left(\zeta_{m}^{+}+\zeta_{m}^{-}\right)=$ $-\frac{1}{2} \ln \left|Z_{m}^{+} Z_{m}^{-}\right|=-\frac{1}{2} \ln \sqrt{1+4 \lambda_{2}^{2}(m \pi)^{2}}$, where $Z_{m}^{ \pm}$stand for the arguments of the logarithm in Eq. (85), does not depend on the penetrability constant $\lambda_{1}$ of the interior barrier. Indeed, simple transformations show that $Z_{m}^{+} Z_{m}^{-}=\left[\left(1-2 i \lambda_{1} m \pi\right) /\left(1+2 i \lambda_{1} m \pi\right)\right]\left(1-2 i \lambda_{2} m \pi\right)$.

We fix now the number $m \gg 1$ within the resonance domain, $\lambda_{1,2}|m \pi| \ll 1$, and consider the doublet of poles 
close to this point. Keeping the terms of the two first orders of magnitude we find

$$
\begin{aligned}
\zeta_{m}^{ \pm} \approx & -\frac{1}{2}\left(2 \lambda_{1}+\lambda_{2} \pm \sqrt{4 \lambda_{1}^{2}+\lambda_{2}^{2}}\right) m \pi \\
& -\frac{i}{2} \lambda_{2}^{2}\left(1 \pm \frac{\lambda_{2}}{\sqrt{4 \lambda_{1}^{2}+\lambda_{2}^{2}}}\right)(m \pi)^{2}
\end{aligned}
$$

In the resonance region, the splitting $\left|\Delta z_{m}\right|=\left|\Delta \zeta_{m}\right| \approx$ $\sqrt{4 \lambda_{1}^{2}+\lambda_{2}^{2}}$ within a given doublet is much smaller than the distance between adjacent doublets which is $\sim \pi$.

Equation (81) reads in the case $L=2$

$$
R(k)=-\frac{2 \lambda_{2} k\left(\sin ^{2} k+\lambda_{1} k \sin 2 k\right)}{\sin ^{2} k+\frac{1}{2}\left(2 \lambda_{1}+\lambda_{2}\right) k \sin 2 k+\lambda_{1} \lambda_{2} k^{2} \cos 2 k} .
$$

For the $m$ th resonance doublet $k_{m}=m \pi+\delta k_{m}$, and the small shift $\delta k_{m}$ satisfies the quadratic equation

$$
\left(\delta k_{m}\right)^{2}+\left(2 \lambda_{1}+\lambda_{2}\right)(m \pi) \delta k_{m}+\lambda_{1} \lambda_{2}(m \pi)^{2}=0,
$$

giving immediately $\delta k_{m}^{ \pm}=\Re \mathrm{e} \zeta_{m}^{ \pm}$, with $\zeta_{m}^{ \pm}$from (86).

This convinces us that each resonance doublet with a good accuracy can be considered independently of the other poles. Near a given $k_{m}=m \pi$ within the resonance region the function $R(m \pi+\delta k) \equiv R_{m}(k)$ is decomposed into a sum of two partial fractions

$$
R_{m}(k)=\frac{\gamma_{m}^{+}}{k-k_{m}^{+}}+\frac{\gamma_{m}^{-}}{k-k_{m}^{-}},
$$

the residues being found to be equal to $\gamma_{m}^{ \pm}=-2 \Im \mathrm{m} \zeta_{m}^{ \pm}$.

To pass from the $k$ to the energy plane, the term $R_{-m}(k)$ must be added, which yields

$$
R_{m}(E)=\frac{\Gamma_{m}^{+}}{E-\varepsilon_{m}^{+}}+\frac{\Gamma_{m}^{-}}{E-\varepsilon_{m}^{-}}=A_{m}^{T} \frac{1}{E-\varepsilon_{m}^{(\mathrm{N})}} A_{m} .
$$

The two-dimensional vector $A_{m}^{T}=\left(\sqrt{\Gamma_{m}^{+}}, \sqrt{\Gamma_{m}^{-}}\right)$and ma$\operatorname{trix} \varepsilon_{m}^{(\mathrm{N})}=\operatorname{diag}\left(\varepsilon_{m}^{+}, \varepsilon_{m}^{-}\right)$of the internal levels $\varepsilon_{m}^{ \pm}=\left(k_{m}^{ \pm}\right)^{2}$ of the doublet have been introduced on the last step.

The corresponding representation of the scattering amplitude near the scattering energy $E \approx k_{m}^{2}$ is as follows:

$$
\tilde{T}_{m}(E)=A_{m}^{T} \frac{1}{E-\left(\mathcal{H}_{\text {eff }}^{(\mathrm{N})}\right)_{m}} A_{m} .
$$

In these formulas $\Gamma_{m}^{ \pm}=2 \sqrt{E} \gamma_{m}^{ \pm} \approx 2 \pi m \gamma_{m}^{ \pm}$, i.e., we have neglected the change of the scattering energy $E$ within the doublet considered. The difference between $k_{m}^{ \pm}$contributes only at higher orders. This is contrary to the case of widths from different doublets when $k_{m}$ differ from each other already in the zero order in $\lambda_{1,2}$. In such an approximation, both functions $R_{m}(E)$ and $\tilde{T}_{m}(E)$ become meromorphic in the complex energy plane.

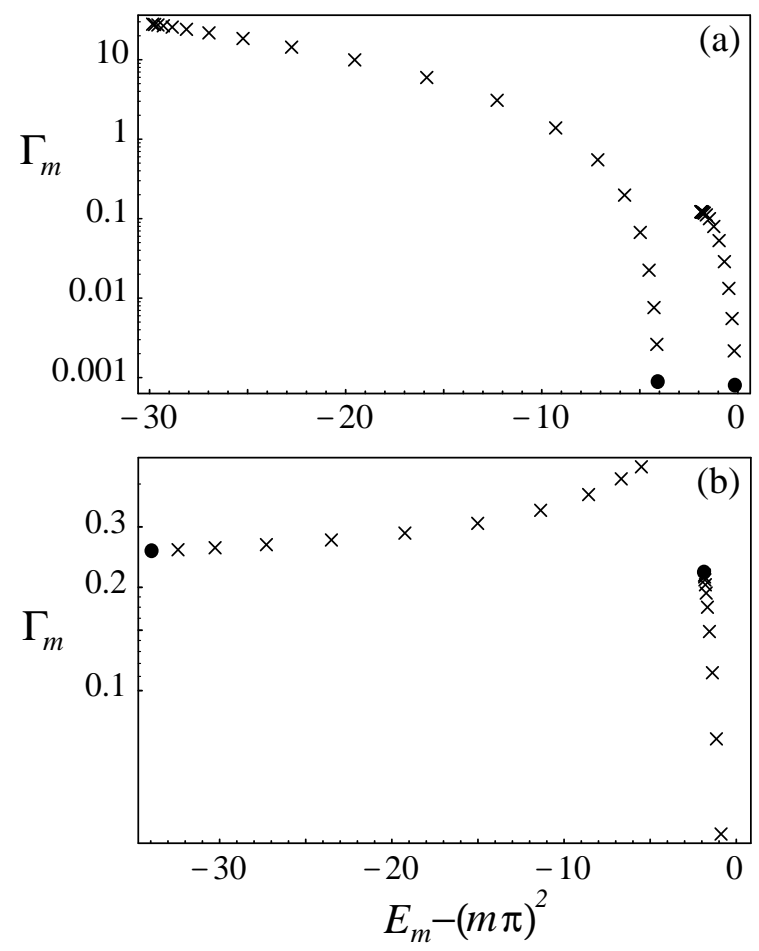

FIG. 3: Widths (in log scale) versus energies for a doublet of resonances with $m=10$ as (a) $\lambda_{1} / \lambda_{2}$ is varied from 0.1 to 10 (marked with $\bullet$ ) at fixed $\lambda_{1}=0.001$; (b) $\lambda_{1} / \lambda_{2}$ is varied from 1 to 10 (marked with $\bullet$ ) at fixed $\lambda_{2}=0.001$.

By the very construction, the complex eigenvalues of the two-dimensional energy-independent symmetric matrix

$$
\mathcal{H}_{\mathrm{eff}}^{(\mathrm{N})}=\varepsilon^{(\mathrm{N})}-\frac{i}{2} \tilde{A} \tilde{A}^{T}
$$

coincide with the complex energies of the two resonances of the doublet considered. Henceforth, we drop the index $m$ of the doublet to avoid too bulky notation. The energies $\varepsilon^{ \pm}$are the levels of the internal problem for the potential $U_{\infty}^{(2)}(r)=\kappa_{1} \delta(r-a)$ with the BC (51) whereas the amplitudes are given by Eq. (58), with the wave number $k$ substituted by $m \pi$. Just the energyindependent matrix (92) is naturally interpreted as an effective non-Hermitian Hamiltonian. The notion of the effective Hamiltonian is valid, however, only within a fixed doublet. Similarly, in the case $L=1$ the "effective Hamiltonian" coincides with the corresponding diagonal matrix element of the formal operator $\mathcal{H}$ constructed in Sec. III.

As we have already mentioned above, the total width $\Gamma=\Gamma_{+}+\Gamma_{-} \approx 4 \lambda_{2}^{2}(m \pi)^{3}$ of the doublet remains constant as long as the openness of the system is fixed. At the same time, the individual widths depend also on the ratio $\lambda_{1} / \lambda_{2}$. A remarkable redistribution of the total width between members of the doublet takes place when this parameter changes. When the system is almost closed, 


$$
\begin{aligned}
& \lambda_{2} \ll \lambda_{1}, \\
& \quad E_{+} \approx\left(1-4 \lambda_{1}-\lambda_{2}\right)(m \pi)^{2}, \quad \Gamma_{ \pm} \approx 2 \lambda_{2}^{2}(m \pi)^{3} . \\
& E_{-} \approx\left(1-\lambda_{2}\right)(m \pi)^{2},
\end{aligned}
$$

Both levels have similar widths in this case. However, in the opposite limit $\lambda_{2} \gg \lambda_{1}$ the state that exists in the outer well appropriates almost the whole total width

$$
\begin{aligned}
E_{+} \approx\left(1-2 \lambda_{2}-2 \lambda_{1}\right)(m \pi)^{2}, & \Gamma_{+} \approx 4\left(\lambda_{2}^{2}-\lambda_{1}^{2}\right)(m \pi)^{3} \\
E_{-} \approx\left(1-2 \lambda_{1}\right)(m \pi)^{2}, & \Gamma_{-} \approx 4 \lambda_{1}^{2}(m \pi)^{3} .
\end{aligned}
$$

Simultaneously, the energy $E_{+}$of the broader resonance gets strongly displaced due to the coupling to the energy continuum. The other resonance turns out to be trapped in the inner well. Figure 3 illustrates the behavior just described. For an arbitrary ratio $\lambda_{1} / \lambda_{2}$, the complex energies of the two resonances of a doublet are separated by a distance which is large on the scale of the total width [35]. For this reason, the off-diagonal matrix elements of the anti-Hermitian part of the effective Hamiltonian (92) give only corrections of higher order and can therefore be neglected. In spite of the nontrivial behavior of the complex levels just described the resonances may still be considered separately of each other within the given accuracy.

Extension to the general case of an arbitrary number $L$ of the barriers is now straightforward. The effective Hamiltonian appears as a $L \times L$ block of the operator $\mathcal{H}$, which describes a fixed bunch of $L$ close resonance states. It is important that energy dependence can be fully neglected within such a bunch.

\section{F. Internal problem with Dirichlet BC}

The internal problem with the BC (51) does not correspond to a finite motion of a quantum particle even when the coupling matrix elements are neglected. Indeed, the internal solution is sensitive to the penetrability of the outer barrier. Meanwhile, in numerous applications the intuitively most attractive convention, which goes back to the textbook 7], is adopted, using as an internal basis the states of a closed counterpart of the system under consideration. Such a counterpart can hardly be defined uniquely but, in our case, it is natural to fix it by choosing Dirichlet BC at the point $r=a$. This corresponds to the internal problem with an infinitely hard wall put at the point $r=a$. The formalism of Sec. II gives then

$$
\begin{aligned}
S(k) & =\frac{1-\frac{i}{\lambda_{\mathrm{L}} k}\left[1+\lambda_{\mathrm{L}} G_{\mathrm{int}}^{(\mathrm{D})^{\prime \prime}}(a, a)\right]}{1+\frac{i}{\lambda_{\mathrm{L}} k}\left[1+\lambda_{\mathrm{L}} G_{\mathrm{int}}^{(\mathrm{D})^{\prime \prime}}(a, a)\right]} S_{0}^{(\mathrm{N})} \\
& =\frac{1+\frac{\lambda_{\mathrm{L}}}{1+i \lambda_{\mathrm{L}} k} G_{\mathrm{int}}^{(\mathrm{D})^{\prime \prime}}(a, a)}{1+\frac{\lambda_{\mathrm{L}}}{1-i \lambda_{\mathrm{L}} k} G_{\mathrm{int}}^{(\mathrm{D})^{\prime \prime}}(a, a)} \frac{1+i \lambda_{\mathrm{L}} k}{1-i \lambda_{\mathrm{L}} k} S_{0}^{(\mathrm{D})} .
\end{aligned}
$$

Comparison with the closed solution (55) and (79) shows that

$$
\cot \left(\delta_{\infty}+k a\right)=\frac{1}{k} G_{\mathrm{int}}^{(\mathrm{D})^{\prime \prime}}(a, a)=\frac{1}{k} \sum_{n} \frac{v_{n}^{0^{\prime}}\left(a_{-}\right) v_{n}^{0^{\prime}}(a)}{k^{2}-\varepsilon_{n}^{(\mathrm{D})}}
$$

which implies that the spectrum of the internal problem is given by the equation $\sin \left(\delta_{\infty}+k a\right)=0$ instead of Eq. (82). Unlike the eigenvalues $\varepsilon_{n}^{(\mathrm{N})}$, the levels $\varepsilon_{n}^{(\mathrm{D})} d o$ not depend on $\lambda_{\mathrm{L}}$.

Similar to Eqs. (55)- (57), we have $S(k)=\bar{S}(k) S_{0}(k)$, with $S_{0}$ from Eq. (54), and $\bar{S}(k)=1-i \bar{A}^{T}\left[k^{2}-\mathcal{H}^{(\mathrm{D})}\right]^{-1} \bar{A}$, where

$$
\begin{aligned}
\mathcal{H}_{m n}^{(\mathrm{D})} & =\varepsilon_{m}^{(\mathrm{D})} \delta_{m n}-\frac{\lambda_{\mathrm{L}}}{1-i \lambda_{\mathrm{L}} k} v_{m}^{0}{ }^{\prime}(a) v_{n}^{0^{\prime}}(a) \\
& =\varepsilon_{m}^{(\mathrm{D})} \delta_{m n}-\frac{1}{2 \lambda_{\mathrm{L}} k} \bar{A}_{m} \bar{A}_{n}-\frac{i}{2} \bar{A}_{m} \bar{A}_{n}
\end{aligned}
$$

and

$$
\bar{A}_{n}=\lambda_{\mathrm{L}} \sqrt{\frac{2 k}{1+\lambda_{\mathrm{L}}^{2} k^{2}}} v_{n}^{0^{\prime}}(a)
$$

This should be compared with Eq. (58) and (59). Not only widths but also Hermitian shifts result from the coupling to the external region this time. Indeed, in the resonance region $\lambda_{\mathrm{L}} k \ll 1$ the phases that come from the first two factors in Eq. (95b) are equal approximately to

$$
\bar{\delta}(k) \approx-\lambda_{\mathrm{L}}^{2} k \frac{G_{\mathrm{int}}^{(\mathrm{D})^{\prime \prime}}(a, a)}{1+\lambda_{\mathrm{L}} G_{\mathrm{int}}^{(\mathrm{D})^{\prime \prime}}(a, a)} ; \quad \delta_{\lambda_{\mathrm{L}}}(k) \approx \lambda_{\mathrm{L}} k .
$$

The two contributions perfectly compensate each other at the points $k_{n}^{2}=\varepsilon_{n}^{(\mathrm{D})}$. Within a bunch of resonance levels the eigenvalues of the Hermitian part of $\mathcal{H}_{\text {eff }}^{(\mathrm{D})}$ coincide in the main approximation with the corresponding levels $\varepsilon_{n}^{(\mathrm{N})}$. In general, the connection $\tilde{\delta}(k)=\bar{\delta}(k)+\delta_{\lambda_{\mathrm{L}}}(k)$ holds for arbitrary $k$.

Let us first return to the case $L=2$, for a moment. The amplitudes (98) for the $m$ th doublet are easily calculated to be in the main approximation $\bar{A}_{ \pm} \approx \pm \sqrt{2} \lambda_{2}(m \pi)^{3 / 2}$, when the corresponding internal levels are $\varepsilon_{+}^{(\mathrm{D})} \approx(1-$ $\left.4 \lambda_{1}\right)(m \pi)^{2}$ and $\varepsilon_{-}^{(\mathrm{D})}=(m \pi)^{2}$. Therefore, the diagonal elements of the effective Hamiltonian of the doublet are equal to

$$
\mathcal{E}_{ \pm}^{(0)} \approx\left\{\begin{array}{l}
\left(1-4 \lambda_{1}-\lambda_{2}\right)(m \pi)^{2}-i \lambda_{2}^{2}(m \pi)^{3} \\
\left(1-\lambda_{2}\right)(m \pi)^{2}-i \lambda_{2}^{2}(m \pi)^{3}
\end{array}\right.
$$

which exactly coincide with Eq. (93). Off-diagonal elements of $\mathcal{H}_{\mathrm{eff}}^{(\mathrm{D})}$ can be neglected if $\lambda_{2} \ll \lambda_{1}$. These elements become important, however, when the coupling to the continuum becomes strong, $\lambda_{2} \gtrsim \lambda_{1}$. The two resonances (99) interfere in this case. Diagonalization of the 
$2 \times 2$-matrix $\mathcal{H}_{\text {eff }}^{(\mathrm{D})}$ gives now

$$
\begin{aligned}
\mathcal{E}_{ \pm} \approx & {\left[1-2 \lambda_{1}-\lambda_{2} \mp \sqrt{4 \lambda_{1}^{2}+\lambda_{2}^{2}}\right](m \pi)^{2} } \\
& -i \lambda_{2}^{2}\left(1 \pm \frac{\lambda_{2}}{\sqrt{4 \lambda_{1}^{2}+\lambda_{2}^{2}}}\right)(m \pi)^{3} .
\end{aligned}
$$

In the limit $\lambda_{2} \gg \lambda_{1}$ this reduces to (94).

For an arbitrary number $L$ of barriers, the $m$ th bunch of $L$ close resonances is described by the $L \times L$-submatrix $\mathcal{H}_{\text {eff }}^{(\mathrm{D})}$ of the infinite matrix (97), with the wave number $k$ being substituted by $k_{m} \approx m \pi$. The absolute values of the coupling amplitudes within such a resonance bunch are estimated as $\left|\bar{A}_{n}\right| \approx 2 \lambda_{\mathrm{L}}(m \pi)^{3 / 2} / \sqrt{L}$. The total collective width $\Gamma_{c}^{(m)}=\sum_{l=1}^{L} \bar{A}_{l}^{2} \equiv \overline{\mathbf{A}}^{2} \approx 4 \lambda_{\mathrm{L}}^{2}(m \pi)^{3}$ of the bunch, which is determined by the trace of the antiHermitian part of $\mathcal{H}_{\mathrm{eff}}^{(\mathrm{D})}$, characterizes the openness of the system and does not depend on the penetrability constant $\lambda_{1}$ of the internal barriers. The same is valid in the main approximation regarding the collective real energy displacement $\delta \epsilon_{c}^{(m)} \equiv 2 \lambda_{\mathrm{L}}(m \pi)^{2} \approx \Gamma_{c}^{(m)} / 2 \lambda_{\mathrm{L}} m \pi$. When this displacement is small in comparison with the total energy spread of the internal levels, $\delta \epsilon_{c}^{(m)} \ll \Delta^{(m)}\left(\varepsilon^{(\mathrm{D})}\right)$, the bunch consists of $L$ independent narrow resonances

$$
\mathcal{E}_{n}^{(m)} \approx \varepsilon_{n}^{(\mathrm{D})}-\frac{1}{L} \delta \epsilon_{c}^{(m)}-\frac{i}{2} \frac{1}{L} \Gamma_{c}^{(m)} .
$$

Note that the Hermitian shift does not influence the level spacings but changes only the position of the bunch.

Under the opposite condition, $\delta \epsilon_{c}^{(m)} \gg \Delta^{(m)}\left(\varepsilon^{(\mathrm{D})}\right)$, the doorway basis in which the interaction matrix $\bar{A} \bar{A}^{T}$ is diagonal becomes more adequate [23] than the basis of the internal problem used up to now (see also [36]). The latter matrix is diagonalized by an orthogonal transformation $\eta=\left(\boldsymbol{\eta}^{(1)} \boldsymbol{\eta}^{(2)} \ldots \boldsymbol{\eta}^{(\mathrm{L})}\right)$, where each entry $\boldsymbol{\eta}^{(l)}$ is a real vector in the $L$-dimensional part of the total Hilbert space, which corresponds to the considered bunch. Due to the factorized structure, the interaction matrix possesses the only nonzero eigenvalue $\Gamma_{c}^{(m)}=\overline{\mathbf{A}}^{2}$, which belongs to the eigenvector $\boldsymbol{\eta}^{(1)}=\mathbf{a} \equiv \overline{\mathbf{A}} / \sqrt{\overline{\mathbf{A}}^{2}}$. As a result, the effective Hamiltonian obtains in the doorway basis the form (we drop below the index $m$ of the bunch)

$$
\mathcal{H}_{\mathrm{eff}}^{(\mathrm{D})}=\left(\begin{array}{cc}
\epsilon_{c}-\frac{i}{2} \Gamma_{c} & \mathbf{h}^{\mathrm{T}} \\
\mathbf{h} & \hat{\epsilon}
\end{array}\right)
$$

The following notation has been used here $(\mu=2,3, \ldots, L)$ :

$$
\begin{aligned}
& \epsilon_{c}=\sum_{l} \varepsilon_{l}^{(\mathrm{D})} a_{l}^{2}-2 \lambda_{\mathrm{L}}(m \pi)^{2}=\left\langle\varepsilon_{l}^{(\mathrm{D})}\right\rangle-\delta \epsilon_{c}, \\
& h_{\mu}=\sum_{l} \varepsilon_{l}^{(\mathrm{D})} a_{l} \eta_{l}^{(\mu)},
\end{aligned}
$$

with the $L-1$ vectors $\boldsymbol{\eta}^{(\mu)}$ and elements $\epsilon_{\mu}$ of the diagonal matrix $\hat{\epsilon}$ being defined by the eigenvalue problem

$$
\sum_{n} \varepsilon_{n}^{(\mathrm{D})} \eta_{n}^{(\mu)} \eta_{n}^{(\nu)}=\epsilon_{\mu} \delta_{\mu \nu}
$$

in the $(L-1)$-dimensional subspace orthogonal to the vector a. The quantity $\left\langle\varepsilon_{l}^{(\mathrm{D})}\right\rangle$ is the weighted mean position of the internal levels. The non-Hermitian Hamiltonian (102) describes a wide doorway resonance with width $\Gamma_{c}$ displaced from the bunch by the distance $\delta \epsilon_{c}$ and coupled to a background of $L-1$ stable states by means of the matrix elements $h_{\mu}$. Only due to this interaction do such states get access to the continuum via the doorway state existing in the outer well.

With the help of the completeness condition

$$
\sum_{\mu} \eta_{l}^{(\mu)} \eta_{l^{\prime}}^{(\mu)}=\delta_{l l^{\prime}}-a_{l} a_{l^{\prime}}
$$

one finds from Eq. (104)

$$
\eta_{l}^{(\mu)}=-h_{\mu} \frac{1}{\epsilon_{\mu}-\varepsilon_{l}^{(\mathrm{D})}} a_{l} .
$$

The orthogonality condition $\mathbf{a} \cdot \boldsymbol{\eta}^{(\mu)}=0$ immediately gives the equation

$$
\sum_{l} \frac{a_{l}^{2}}{\epsilon_{\mu}-\varepsilon_{l}^{(\mathrm{D})}}=0
$$

for the new positions of the stable levels. This equation shows that each new level $\epsilon_{\mu}$ lies between two neighboring old ones $\varepsilon_{l}^{(\mathrm{D})}$ and therefore is shifted with respect to the latter only by a distance comparable with the initial mean level spacing. This is much smaller than the displacement $\delta \epsilon_{c}$ of the collective level $\epsilon_{c}$.

The interaction $\mathbf{h}$ mixes these states and forms $L$ final resonance states. The complex energies of the exact states, i.e. the eigenvalues of the effective Hamiltonian (102), satisfy the secular equation

$$
\mathcal{E}=\epsilon_{c}-\frac{i}{2} \Gamma_{c}+\sum_{\mu} \frac{h_{\mu}^{2}}{\mathcal{E}-\epsilon_{\mu}}
$$

In particular, for the collective doorway state one obtains from this equation

$$
\mathcal{E}_{\text {coll }} \approx\left\langle\varepsilon_{l}^{(\mathrm{D})}\right\rangle-\left[1-\frac{\mathbf{h}^{2}}{\left(\delta \epsilon_{c}\right)^{2}}\right] \delta \epsilon_{c}-\frac{i}{2}\left[1-\frac{\mathbf{h}^{2}}{\left(\delta \epsilon_{c}\right)^{2}}\right] \Gamma_{c} .
$$

Equations (103) and (105) allow us to express the square length $\mathbf{h}^{2}$ of the mixing vector in terms of the variance of the non-perturbed internal levels,

$$
\begin{aligned}
\mathbf{h}^{2} & =\sum_{l}\left(\varepsilon_{l}^{(\mathrm{D})}\right)^{2} a_{l}^{2}-\left(\sum_{l} \varepsilon_{l}^{(\mathrm{D})} a_{l}^{2}\right) \\
& =\left\langle\left(\varepsilon^{(\mathrm{D})}-\left\langle\varepsilon^{(\mathrm{D})}\right\rangle\right)^{2}\right\rangle=\left[\Delta \varepsilon^{(\mathrm{D})}\right]^{2} .
\end{aligned}
$$

Thus, interaction is weak in the doorway basis under the condition $\delta \epsilon_{c} \gg \Delta \varepsilon^{(\mathrm{D})}$ and the doorway state keeps almost the whole energy displacement and width. All other states are trapped in the interior region and share small 
portions $\sim\left[\Delta \varepsilon^{(\mathrm{D})} / \delta \epsilon_{c}\right]^{2}$ of the collective displacement and width in accordance with the group velocity [23] . The solution just described has formally very much in common with the schematic model of the so-called nuclear dipole giant resonance developed in [36, 37].

\section{CONCLUSION}

In this paper we analyzed the relevance of the concept of the non-Hermitian effective Hamiltonian in finiterange potential scattering. Single-channel $s$-wave resonance scattering is considered as an example. The scattering function $S(k)$ is meromorphic in this case, i.e., has only isolated poles in the complex plane of the wave numbers. The number of poles is, generally, infinite but only a finite part of them can be interpreted as resonances.

We first presented (Secs. II and III) a consistent selfadjoint formulation of the scattering problem, which is based on separation of the configuration space into internal and external segments. In this way, the $S(k)$ function is represented in terms of a non-Hermitian energydependent operator $\mathcal{H}(k)$. There exists a wide freedom in choosing the radius $a$ of separation as well as the boundary conditions at this point. Different choices yield different explicit forms of this operator together with the $S(k)$ and $R(k)$ functions. This can, in particular, come out strongly if one truncates the matrix $\mathcal{H}$ to calculate numerically the positions of the poles of $S$ function in the complex $k$ plane. Nevertheless, the true complex poles of the $S$ function and this function itself depend, as we explicitly demonstrate, neither on the $\mathrm{BC}$ nor on the radius $a$.

Although all choices are formally allowed, this does not mean that they are equally adequate from the physical point of view. For instance, a fictitious immediate reflection takes place at the (arbitrarily chosen) point of separation $a$. The artificial separation of the phase of this reflection results, in turn, in the appearance of an infinite number of remote poles of the function $R(k)$, which describe the smooth contribution from the interior region $r<a$. The phase of the immediate reflection must be compensated by the contributions of such poles for the radius $a$ to disappear finally from the scattering amplitude. This can cause unjustified complications in intermediate stages of calculations. We argue that the harm is minimized if the separation radius matches an outer potential barrier when it is strong enough to make immediate reflection at this point quite probable.

The notion of the non-Hermitian effective Hamiltonian first introduced in the theory of resonance nuclear reactions emerges when a group of very close resonance states strongly overlap and interfere. We stress that, in contrast to the nuclear reactions, in the cases of the potential resonance scattering usually discussed in the literature the density of the energy spectrum is too low and the ordinary Breit-Wigner approximation of isolated resonances usually suffices. The complex energy of the $m$ th resonance which dominates in the scattering amplitudes near the scattering energy $E \approx\left|z_{m}\right|^{2}$ is well approximated by the diagonal matrix element $\mathcal{H}_{m m}\left(\left|z_{m}\right|\right)$. There is no room for an effective Hamiltonian in this case or, more strictly, it is embodied by the $1 \times 1$ "matrix" $\mathcal{H}_{m m}$. We emphasize that the energy dependent operator $\mathcal{H}(k)$ should not be confused with the effective Hamiltonian. Energy dependent eigenvalues of this operator are not, generally, in one-to-one correspondence with the complex energies of the actual resonance states.

Overlap and interference of the resonances can become possible in the cases when the energy spectrum has a band structure. As an example of this kind we investigate a periodically disposed chain of a finite number $L$ of radial $\delta$ barriers. All $S, K$ and $R$ functions are found in closed forms in terms of an $L \times L k$-dependent matrix propagator. This allows us to study in detail all analytical properties in the complex $k$ plane and to verify the correspondence with the projection formalism used. There exist a finite number of separated bands of close resonances. Within the $m$ th band which lie near the scattering energy $E \approx\left|z_{m}\right|^{2}$ one can neglect all smooth variations with $k$. In this approximation, the mentioned propagator proved to coincide with the resolvent of the $L \times L$ block $\mathcal{H}_{l l^{\prime}}\left(\left|z_{m}\right|\right)$ of the matrix $\mathcal{H}_{m n}^{(\mathrm{N})}(k)$ taken at the fixed value $k=\left|z_{m}\right|$. Just this matrix plays the role of the effective Hamiltonian of the system in the energy interval within the band.

Different choices of BCs yield different patterns of the resonance interference. In particular, the spectrum of the poles of the $R$ function is exactly reproduced in the framework of the projection technique with the BC (51) of Neumann type fixed at the position $a=L$ of the outer barrier. The corresponding energy levels depend on the penetrability constant $\lambda_{\mathrm{L}}$ of the outer barrier. Shifts of the levels due to the coupling to the continuum are included in this case from the very beginning.

Utilization of the Dirichlet BC for the intrinsic motion gives another but yet equivalent formulation of the scattering problem considered. The internal problem in this case fixes a closed counterpart of the open system under consideration. This enables us to investigate the change of the regime of the internal motion of the system as its openness grows. The interaction via the continuum shifts the original $\lambda_{\mathrm{L}}$-independent levels of the internal motion along both real and imaginary axes. A transition is explicitly demonstrated from the bunch of $L$ similar narrow resonances to the formation of a relatively broad resonance strongly shifted with respect to the band, which exists in the outer well. The other resonances turn out to be trapped in the inner part of the system. This is quite similar to the results of earlier investigations which rely upon the notion of the non-Hermitian effective Hamiltonian of an open system. 


\section{Acknowledgments}

We are grateful to Y.V. Fyodorov for discussions on Feshbach's projector technique in an early stage of this work, to H. Schanz and V. Zelevinsky for interesting discussions, and to G. Hackenbroich and C. Viviescas for their interest in the work and useful conversations. One of us (V.V.S.) greatly appreciates the generous hospitality of the Max-Planck Institute for the Complex Systems extended to him during his stay in Dresden. The financial support by RFBR Grant No. 03-02-16151 (D.V.S. and V.V.S.) and SFB 237 "Unordnung and grosse Fluktuationen" (D.V.S. and H.J.S.) is acknowledged with thanks.
[1] H. Feshbach, Ann. Phys. (N.Y.) 5, 357 (1958).

[2] H. Feshbach, Ann. Phys. (N.Y.) 19, 287 (1962), [reprinted in Ann. Phys. (N.Y.) 281, 519 (2000)].

[3] P. L. Kapur and R. Peierls, Proc. R. Soc. London, Ser. A 166, 277 (1938).

[4] E. Wigner and L. Eisenbud, Phys. Rev. 72, 29 (1947).

[5] M. S. Livšic, Sov. Phys. JETP 4, 91 (1957).

[6] M. S. Livšic, Operators, Oscillations, Waves (Open Systems), Translations of Mathematical Monographs Vol. 34 (American Mathematical Society, Providence, RI, 1973).

[7] C. Mahaux and H. A. Weidenmüller, Shell-Model Approach to Nuclear Reactions (North-Holland, Amsterdam, 1969).

[8] J. J. M. Verbaarschot, H. A. Weidenmüller, and M. R. Zirnbauer, Phys. Rep. 129, 367 (1985).

[9] V. V. Sokolov and V. G. Zelevinsky, Nucl. Phys. A 504, 562 (1989).

[10] F. Remacle, M. Munster, V. B. Pavlov-Verevkin, and M. Desouter-Lecomte, Phys. Lett. A 145, 265 (1990); M. Desouter-Lecomte and F. Remacle, Chem. Phys. 164, 11 (1992).

[11] I. Rotter, Rep. Prog. Phys. 54, 635 (1991).

[12] Y. V. Fyodorov and H.-J. Sommers, J. Math. Phys. 38, 1918 (1997).

[13] F.-M. Dittes, Phys. Rep. 339, 215 (2000).

[14] Y. Alhassid, Rev. Mod. Phys. 72, 895 (2000).

[15] H.-J. Stöckmann, Quantum Chaos: An Introduction (Cambridge University Press, Cambridge, UK, 1999).

[16] G. Akguc and L. E. Reichl, Phys. Rev. E 64, 056221 (2001).

[17] K. Pichugin, H. Schanz, and P. Šeba, Phys. Rev. E 64, 056227 (2001).

[18] H.-J. Stöckmann, E. Persson, Y.-H. Kim, M. Barth, U. Kuhl, and I. Rotter, Phys. Rev. E 65, 066211 (2002).

[19] G. Hackenbroich, C. Viviescas, and F. Haake, Phys. Rev. Lett. 89, 083902 (2002); C. Viviescas and G. Hackenbroich, Phys. Rev. A 67, 013805 (2003).

[20] H. Alt, P. von Brentano, H.-D. Gräf, R.-D. Herzberg, M. Philipp, A. Richter, and P. Schardt, Nucl. Phys. A 560, 293 (1993).

[21] H. Alt, H.-D. Gräf, H. L. Harney, R. Hofferbert, H. Lengeler, A. Richter, P. Schardt, and H. A. Weidenmüller, Phys. Rev. Lett. 74, 62 (1995).

[22] E. Persson, I. Rotter, H.-J. Stöckmann, and M. Barth,
Phys. Rev. Lett. 85, 2478 (2000)

[23] V. V. Sokolov and V. G. Zelevinsky, Ann. Phys. (N.Y.) 216, 323 (1992).

[24] T. Kottos and U. Smilansky, Ann. Phys. (N.Y) 274, 76 (1999).

[25] F. Barra and P. Gaspard, J. Phys. A: Math. Gen. 32, 3357 (1999). We are grateful to T. Kottos for drawing our attention to this paper.

[26] C. Bloch, Nucl. Phys. 4, 503 (1957).

[27] A. M. Lane and D. Robson, Phys. Rev. 151, 774 (1966); D. Robson and A. M. Lane, ibid. 161, 982 (1967).

[28] A. M. Lane and R. G. Thomas, Rev. Mod. Phys. 30, 257 (1958).

[29] As usual, factors $e^{-\alpha n}$ with infinitesimal $\alpha$ should be introduced to guarantee the absolute convergence.

[30] Let us recall that the penetrability of a $\delta$-barrier $D(k)=$ $(\lambda k)^{2} /\left[1+(\lambda k)^{2}\right]$ is small, $D(k) \approx(\lambda k)^{2}$, if $\lambda k \ll 1$, and goes to one under the opposite condition.

[31] N. Hu, Phys. Rev. 74, 131 (1948).

[32] H. M. Nussenzveig, Causality and Dispersion Relations (Academic Press, New York, 1972).

[33] E. Persson, M. Müller, and I. Rotter, Phys. Rev. C 53, 3002 (1996).

[34] V. Zelevinsky and A. Volya, in Challenges of Nuclear Structure, edited by A. Covello (World Scientific, Singapore, 2002), p. 261; see also A. Volya and V. Zelevinsky, e-print nucl-th/0211039

[35] By a slight shift of the position of the outer barrier and subsequent careful tuning of the two free parameters complete degeneracy of the resonances can be achieved; see J. S. Bell and C. J. Goebel, Phys. Rev. 138, B 1198 (1965); E. Hernández, A. Jáuregui, and A. Mondragón, J. Phys. A: Math. Gen. 33, 4507 (2000). Recently, the experimental study of such an exceptional point has been performed in a microwave cavity setup: C. Dembowski, H.-D. Gräf, H. L. Harney, A. Heine, W. D. Heiss, H. Rehfeld, and A. Richter, Phys. Rev. Lett. 86, 787 (2001); see, however, I. Rotter, Phys. Rev. E 67, 026204 (2003).

[36] V. V. Sokolov, I. Rotter, D. V. Savin, and M. Müller, Phys. Rev. C 56, 1031 (1997).

[37] V. V. Sokolov and V. G. Zelevinsky, Fizika (Zagreb) 22, 303 (1990). 